\title{
EL DESEMPEÑO DE LOS FONDOS DE INVERSIÓN EN BOLIVIA (2012-2016)
}

\author{
Alejandro Vargas Sanchez ${ }^{1}$ \\ Recibido: 17/03/2017 \\ Aprobado: 17/02/2018
}

\begin{abstract}
RESUMEN
Uno de los temas centrales en el proceso de gestión de portafolios es evaluar la rentabilidad y el riesgo de una inversión. Este documento presenta por primera vez una medición del desempeño alcanzado por los fondos de inversión en Bolivia. La investigación utiliza información pública obtenida de la Autoridad de Supervisión del Sistema Financiero para el periodo 2012 a 2016. El método elegido se basa en la aplicación de indicadores de desempeño y en la estimación econométrica del modelo de mercado, de esta manera se grafican los fondos de inversión en función a su nivel de equilibrio con respecto a la Línea del Mercado de Títulos. En base a los resultados, se logra identificar a los fondos de inversión que consiguieron el mejor desempeño.
\end{abstract}

PALABRAS CLAVE: INVERSIÓN, ESTRUCTURA DE MERCADO, FINANCIACIÓN, MERCADO FINANCIERO

CLASIFICACIÓN JEL: E12, E31, E52

\begin{abstract}
One of the central issues in the portfolio management process, is the task of evaluating the return and risk of an investment. This paper presents for the first time a measurement of the performance achieved by the Mutual Funds in Bolivia. The research uses public information obtained from the Supervision Authority of the Financial System for the period 2012 to 2016 . The chosen method is based on the application of performance indicators and the econometric estimation of the market model, in this way the Mutual Funds are plotted according to their equilibrium level stated by the Securities Market Line. Based on the results, it was possible to identify the Mutual Funds that accomplish the best performance.
\end{abstract}

KEYWORDS: INVESTMENT, MARKET STRUCTURE, FINANCING, FINANCIAL MARKETS. JEL CLASIFICATION: E12, E31, E52

1 Universidad Privada Boliviana (UPB), Centro de Investigación e Innovación en Finanzas (CIIFI); La Paz, Bolivia; alejandrovargas@ lp.upb.edu. 


\section{INTRODUCCIÓN}

El análisis del desempeño de una inversión debería ser una práctica permanente en la gestión de portafolios. Invertir implica tomar decisiones que tienen consecuencias que pueden ser evaluadas. Como señalan Bailey, Richards y Tierney (2016), la evaluación del desempeño tiene tres componentes $^{2}$ : medición del desempeño, atribución del desempeño y estimación del desempeño. La presente investigación tiene como objetivo principal la medición del desempeño alcanzado por los Fondos de Inversión en Bolivia y la estimación del desempeño, mediante el modelo de mercado y la Línea del Mercado de Títulos.

En ese marco, el presente trabajo se desarrolla en cuatro partes: primero la introducción; luego en la segunda parte, se presenta la metodología, donde se describen los indicadores de medición desempeño y se presenta el modelo de equilibrio seleccionado; en la tercera parte se exponen los resultados de la investigación, mediante el cálculo de los indicadores mencionados y la estimación del modelo de mercado, a partir del cual se realiza la comparación respecto a la Línea del Mercado de Títulos; finalmente, en la cuarta parte se presentan las conclusiones del trabajo.

\section{Descripción de los fondos de inversión}

En Bolivia existen un total de 11 Sociedades Administradoras de Fondos de Inversión (SAFI) ${ }^{3}$; desde enero de 1997 hasta el 31 de diciembre de 2016 se han registrado un total de 77 fondos de inversión, de los cuales se encuentran vigentes 64: 35 son Fondos de Inversión Abiertos (FIA) y 29 son Fondos de Inversión Cerrados (FIC). Se destaca la participación de FORTALEZA SAFI S.A. que tiene un total de 16 fondos ( $25 \%$ del total), como se puede apreciar en el cuadro 1.

Los fondos de inversión están denominados en una de tres monedas: bolivianos, dólares americanos y Unidad de Fomento a la Vivienda ${ }^{4}$ (UFV). Al 31 de diciembre de 2016, los FIA se componen de 16 fondos en bolivianos, 18 fondos en dólares y un fondo en UFV; por otro lado, los FIC se componen de 22 fondos en bolivianos y 7 fondos en dólares. Al tratarse de fondos expresados en tres diferentes monedas, para homogeneizar los datos, el análisis y la exposición de todos los resultados fueron expresados en bolivianos, que constituye la moneda de curso legal en Bolivia.

La Autoridad de Supervisión del Sistema Financiero ASFI (2017) publica datos históricos de cada fondo de inversión, con una frecuencia diaria que incluye: la serie, fecha, valor de cada cuota, cuotas vigentes, número de participantes, tasas del último día y de los últimos 30, 90, 180 y 360 días, tasa efectiva anual, total liquidez, cartera neta, cartera bruta y moneda del fondo. La cartera

2 En inglés se denomina performance evaluation y se compone de: performance measurement, performance attribution $y$ performance appraisal.

3 En concordancia con la Ley del Mercado de Valores No. 1834, "un fondo de inversión, es un mecanismo de inversión compuesto por los aportes de varios participantes, ya sean personas jurídicas o naturales, quienes forman un patrimonio común y autónomo. Este patrimonio es administrado por una Sociedad Administradora, misma que realiza inversiones en valores emitidos tanto a nivel nacional como internacional, por cuenta y riesgo de los participantes. El rendimiento de estas inversiones es distribuido proporcionalmente entre todos los participantes del Fondo. Los fondos de inversión pueden ser abiertos o cerrados. Los fondos abiertos se caracterizan por tener un patrimonio variable en el que las cuotas de participación colocadas entre el público son redimibles o rescatables directamente por el fondo en cualquier momento, siendo su plazo de duración indefinido. Los fondos cerrados se caracterizan por tener un patrimonio inicial determinado y una duración previamente establecida, siendo sus cuotas de participación colocadas entre el público no redimibles o rescatables directamente por el fondo, salvo en las circunstancias y procedimientos dispuestos específicamente por sus reglamentos internos" (SAFI BISA, 2018, párr. 1-4).

4 De acuerdo al Banco Central de Bolivia (2017c), "La Unidad de Fomento de Vivienda (UFV) es un índice referencial que muestra la evolución diaria de los precios y se calcula sobre la base del Índice de Precios al Consumidor (IPC) que publica el Instituto Nacional de Estadística (INE). La UFV fue creada mediante el Decreto Supremo 26390 de 8 de noviembre de 2001 y, por Resolución de Directorio del Banco Central de Bolivia No 116/2001 de 20 de noviembre de 2001, se reglamenta su cálculo". 
CUADRO 1

SAFIS EN BOLIVIA Y SUS TIPOS DE FONDOS (DICIEMBRE 2016)

\begin{tabular}{lrrr}
\hline \multicolumn{1}{c}{ NOMBRE SAFI } & FIA & FIC & Sub Total \\
\hline ALIANZA SAFI S.A. & & 1 & 1 \\
BISA SAFI S.A. & 7 & 2 & 6 \\
BNB SAFI S.A. & & 2 & 9 \\
CAPITAL + SAFI S.A. & 4 & 3 & 3 \\
CREDIFONDO SAFI S.A. & 8 & 8 & 4 \\
FORTALEZA SAFI S.A. & & 2 & 16 \\
MARCA VERDE SAFI S.A. & 5 & 2 & 2 \\
MERCANTIL SANTA CRUZ SAFI S.A. & & 3 & 7 \\
PANAMERICAN SAFI S.A. & 5 & 2 & 3 \\
SAFI UNION S.A. & 2 & 4 & 7 \\
SANTA CRUZ INVESTMENT SAFI S.A. & 35 & 29 & 64 \\
Total general & & & 6
\end{tabular}

Fuente: Elaboración propia en base a datos publicados por la ASFI

bruta de cada fondo se compone de las inversiones en títulos valores, la liquidez y otros ingresos y gastos; para hallar la cartera neta se debe deducir de la cartera bruta las comisiones. El valor de las inversiones dependerá del tipo de instrumento, que puede ser local o internacional en función de la política de inversión de cada fondo contenida en su Reglamento. El valor de cada instrumento está afectado por las negociaciones ocurridas en mercado primario o secundario en la Bolsa Boliviana de Valores, para instrumentos locales, o en bolsas internacionales para inversiones en el exterior. Los precios de mercado se determinan mediante la aplicación de una metodología de valoración específica, publicada por la ASFI (2018) que debe utilizar cada fondo.

El valor de la cartera neta y el número de fondos de inversión constituidos por las SAFI reflejan la dinámica que ha tenido el sector a lo largo de 20 años ${ }^{5}$. El gráfico 1 muestra que la serie de tiempo se inició con el año 1997, cuando se crearon un total de 9 fondos de inversión, con un valor total de la cartera neta de 600 millones de bolivianos ${ }^{6}$. Más adelante, en el año 2016, se contaba ya con 64 fondos de inversión, con un valor total en cartera neta que supera los 18.1 mil millones de bolivianos. Al inicio, la mayoría de los fondos de inversión fueron de tipo abiertos en dólares, posteriormente se incrementó el número de fondos en bolivianos, aspecto que respondió al proceso de "bolivianización" de la economía iniciado el año 20047. Otro aspecto a destacar es que, a partir del año 2007, se incrementó en el número de Fondos de Inversión Cerrados principalmente aquellos denominados en bolivianos.

5 También se calculó el coeficiente de correlación, entre la tasa de crecimiento real del PIB y la tasa de crecimiento real de la cartera neta total de los Fondos (se utilizó el deflactor implícito del PIB publicado por el Instituto Nacional de Estadística para hallar los valores reales). Los resultados encontrados fueron concordantes a nivel agregado con aquellos presentados por Humerez y Yañez (2011), no existiendo una relación entre estas variables, ya que el coeficiente de correlación es de -1\% y no es estadísticamente significativo.

6 Todos los fondos en dólares y UFV se encuentran expresado en bolivianos al tipo de cambio vigente en su respectivo periodo.

7 Como menciona Gonzales (2011), la bolivianización se define como el aumento del uso de moneda local como medio de pago, depósito de valor o unidad de cuenta. 


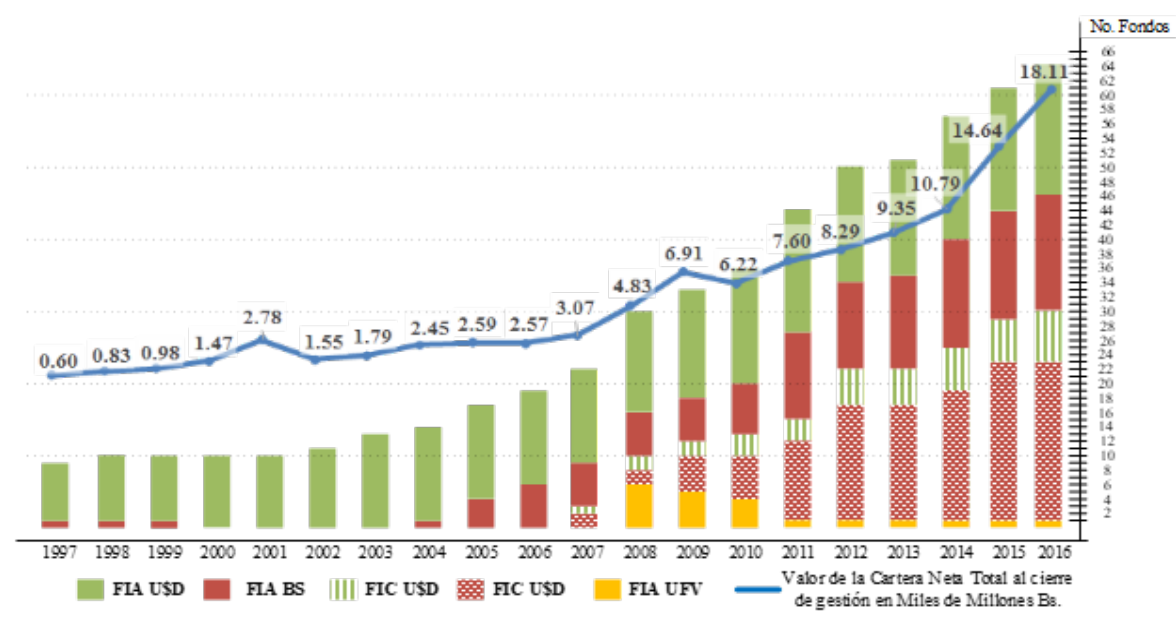

Fuente: Elaboración propia con datos de la evaluación PISA 2012.

Se realizó una aproximación de la estructura de mercado en la que compiten los fondos, por tipo de moneda. En el mercado correspondiente a los fondos en bolivianos, de acuerdo al Índice Herfindahl-Hirschman, tanto los fondos abiertos como los fondos cerrados presentaron un nivel de concentración moderado, con valores de 1.686 y $1.685^{8}$ respectivamente. De acuerdo al número de participantes y el valor total en cartera bruta, las SAFI abiertas BNB, Mercantil SC y Credifondo fueron las más importantes; por otro lado, en términos del número de participantes Mercantil SC, BNB y Fortaleza son las que tuvieron mayor alcance. En el caso de las SAFI cerradas, Santa Cruz Investment, Fortaleza y Capital + son las que tuvieron mayor número de fondos y cartera bruta administrada, en número de participantes no existen importantes diferencias, toda vez que en la mayor parte de los casos se tratan de inversionistas institucionales. Toda esta información se presenta en el cuadro 2. 
CUADRO 2

FONDOS EN BOLIVIANOS, CARTERA BRUTA Y NÚMERO DE PARTICIPANTES, AL 31 DE DICIEMBRE DE 2016

\begin{tabular}{lrrrrr}
\hline & $\begin{array}{c}\text { Número } \\
\text { de } \\
\text { Fondos }\end{array}$ & $\begin{array}{c}\text { Cartera } \\
\text { Bruta Total } \\
\text { Bs. }\end{array}$ & $\begin{array}{c}\text { Número } \\
\text { Total de } \\
\text { Partici- } \\
\text { pantes }\end{array}$ & $\begin{array}{c}\text { Cartera } \\
\text { Bruta } \\
\text { Promedio } \\
\text { Bs. }\end{array}$ & $\begin{array}{c}\text { Promedio } \\
\text { de Partici- } \\
\text { pantes }\end{array}$ \\
\hline FIA & 16 & 4.903 .318 .634 & 34.087 & 306.457 .415 & 2.130 \\
BISA & 2 & 695.174 .702 & 3.260 & 347.587 .351 & 1.630 \\
BNB & 4 & 939.094 .858 & 7.679 & 234.773 .714 & 1.920 \\
CREDIFONDO & 2 & 862.639 .032 & 1.548 & 431.319 .516 & 774 \\
FORTALEZA & 2 & 820.085 .799 & 5.474 & 410.042 .899 & 2.737 \\
MERCANTIL SC & 2 & 881.926 .062 & 11.164 & 440.963 .031 & 5.582 \\
SANTA CRUZ INVESTMENT & 1 & 2.179 .416 & 45 & 2.179 .416 & 45 \\
UNION & 3 & 702.218 .765 & 4.917 & 234.072 .922 & 1.639 \\
FIC & 18 & 7.905 .381 .763 & 69 & 439.187 .876 & 4 \\
ALIANZA & 1 & 588.270 .443 & 4 & 588.270 .443 & 4 \\
BISA & 1 & 436.806 .103 & 3 & 436.806 .103 & 3 \\
CAPITAL + & 3 & 1.598 .231 .484 & 10 & 532.743 .828 & 3 \\
FORTALEZA & 3 & 1.090 .225 .890 & 12 & 363.408 .630 & 4 \\
MARCA VERDE & 1 & 220.003 .501 & 5 & 220.003 .501 & 5 \\
MERCANTIL SC & 1 & 168.171 .705 & 3 & 168.171 .705 & 3 \\
PANAMERICAN & 3 & 908.090 .731 & 11 & 302.696 .910 & 4 \\
SANTA CRUZ INVESTMENT & 3 & 2.207 .861 .298 & 8 & 735.953 .766 & 3 \\
UNION & 2 & 687.720 .610 & 13 & 343.860 .305 & 7
\end{tabular}

Fuente: Elaboración propia en base a datos publicados por la ASFI

Para el mercado de los fondos en dólares, el Índice Herfindahl-Hirschman tanto en los Fondos Abiertos como los Fondos Cerrados mostraron un nivel de concentración alto, con valores de 2.106 y 3.326 respectivamente. Tomando el número de participantes y el valor total en cartera bruta, las SAFI abiertas BNB, Mercantil SC y Fortaleza fueron las más importantes. En el caso de las SAFI cerradas, BNB, Marca Verde y BISA son las que tuvieron mayor cartera bruta administrada. Toda esta información se puede apreciar en el cuadro 3. 
CUADRO 3

FONDOS EN DÓLARES, CARTERA BRUTA Y NÚMERO DE PARTICIPANTES, AL 31 DE DICIEMBRE DE 2016

\begin{tabular}{|c|c|c|c|c|c|}
\hline SAFI & $\begin{array}{l}\text { Número } \\
\text { de } \\
\text { Fondos }\end{array}$ & $\begin{array}{c}\text { Cartera } \\
\text { Bruta Total } \\
\text { Bs. }\end{array}$ & $\begin{array}{l}\text { Número } \\
\text { Total de } \\
\text { Partici- } \\
\text { pantes }\end{array}$ & $\begin{array}{c}\text { Cartera } \\
\text { Bruta } \\
\text { Promedio } \\
\text { Bs. }\end{array}$ & $\begin{array}{c}\text { Promedio } \\
\text { de Partici- } \\
\text { pantes }\end{array}$ \\
\hline FIA & 17 & 3.877 .393 .884 & 36.090 & 228.081 .993 & 2.123 \\
\hline BISA & 2 & 615.400 .406 & 3.192 & 307.700 .203 & 1.596 \\
\hline BNB & 2 & 963.548 .451 & 10.249 & 481.774 .226 & 5.125 \\
\hline CREDIFONDO & 2 & 295.253 .395 & 1.810 & 147.626 .698 & 905 \\
\hline FORTALEZA & 5 & 620.791 .488 & 5.694 & 124.158 .298 & 1.139 \\
\hline MERCANTIL SC & 3 & 1.155 .936 .282 & 13.117 & 385.312 .094 & 4.372 \\
\hline SANTA CRUZ INVESTMENT & 1 & 3.239 .219 & 4 & 3.239 .219 & 4 \\
\hline UNION & 2 & 223.224 .643 & 2.024 & 111.612 .321 & 1.012 \\
\hline FIC & 6 & 1.694 .908 .713 & 36 & 282.484 .786 & 6 \\
\hline BISA & 1 & 235.532 .834 & 4 & 235.532 .834 & 4 \\
\hline BNB & 2 & 789.751 .934 & 13 & 394.875 .967 & 7 \\
\hline FORTALEZA & 1 & 90.993 .278 & 4 & 90.993 .278 & 4 \\
\hline MARCA VERDE & 1 & 513.709 .153 & 2 & 513.709 .153 & 2 \\
\hline MERCANTIL SC & 1 & 64.921 .514 & 13 & 64.921 .514 & 13 \\
\hline
\end{tabular}

Fuente: Elaboración propia en base a datos publicados por la ASFI

Finalmente, se debe mencionar que solo existe un fondo de inversión abierto en UFV que es administrado por FORTALEZA SAFI, el cual, al 31 de diciembre de 2016 tiene una cartera bruta de 295.629.243 Bs. con 5.974 participantes.

\section{METODOLOGÍA}

El propósito de esta sección es proporcionar una visión general de las técnicas y modelos de medición del desempeño, centrándose en cómo los inversores institucionales llevan a cabo la evaluación de los activos invertidos en sus portafolios de inversión.

\section{Indicadores de medición del desempeño}

Como señala Bacon (2013), la medición del desempeño es un proceso científico que consiste en comparar un patrón seleccionado con determinados estándares. Este proceso tiene una naturaleza bidimensional; ya que por un lado se mide la rentabilidad generada por un gestor de portafolios durante un periodo $y$, por otro lado, se cuantifica el riesgo incurrido a lo largo de este mismo periodo; por tanto, el retorno como la forma de la distribución de retorno son de interés para los inversores. 
Para realizar la medición, se necesitan estadísticas descriptivas que ayuden a entender la distribución subyacente de los retornos, así como medidas compuestas de riesgo que determinen si el riesgo asumido por el gestor de cartera se ha justificado por la recompensa recibida. En ese contexto, en el cuadro 4 se plantean 19 indicadores que fueron utilizados para la evaluación del desempeño de cada uno de los fondos de inversión.

CUADRO 4

INDICADORES PARA LA MEDICIÓN DEL DESEMPEÑO

\begin{tabular}{|c|c|c|c|}
\hline No. & Indicador & Descripción & Formula \\
\hline 1 & $\begin{array}{l}\text { Promedio aritmético } \\
\text { mensual }\end{array}$ & $\begin{array}{l}\text { Promedio aritmético simple } \\
\text { de los rendimientos obtenidos } \\
\text { en un mes, considerando un } \\
\text { periodo de } 30 \text { días. }\end{array}$ & $\begin{array}{l}r_{m}=\left[\prod_{t=1}^{30}\left(1+r_{t}\right)\right]-1 \\
\text { Donde: } \\
r_{m} \text { Rendimiento mensual } \\
\bar{r}_{M}=\frac{\sum_{m=1}^{M} r_{m}}{M} \\
\text { Donde: } \\
\bar{r}_{M} \text { Rendimiento promedio } \\
\text { mensual, en base al } \\
r_{m} \text { correspondiente a fin de mes. } \\
M \text { es el número de meses en el } \\
\text { periodo. }\end{array}$ \\
\hline 2 & $\begin{array}{l}\text { Promedio aritmético } \\
\text { anual }\end{array}$ & $\begin{array}{l}\text { Promedio aritmético simple } \\
\text { de los rendimientos obtenidos } \\
\text { en un año, considerando un } \\
\text { periodo de } 360 \text { días. }\end{array}$ & $\begin{array}{l}r_{a}=\left[\prod_{t=1}^{360}\left(1+r_{t}\right)\right]-1 \\
\text { Donde: } \\
r_{a} \text { Rendimiento anual } \\
\bar{r}_{A}=\frac{\sum_{m=1}^{A} r_{a}}{A} \\
\text { Donde: } \\
\bar{r}_{A} \text { Rendimiento promedio anual, } \\
\text { en base al } \\
r_{a} \text { correspondiente a fin de año. } \\
\text { A es el número de años en el } \\
\text { periodo }\end{array}$ \\
\hline
\end{tabular}




\begin{tabular}{|c|c|c|c|c|}
\hline No. & Indicador & Descripción & Formulic & \\
\hline 3 & $\begin{array}{l}\text { Rentabilidad } \\
\text { anualizada }\end{array}$ & $\begin{array}{l}\text { Promedio geométrico de los } \\
\text { rendimientos obtenidos en un } \\
\text { año, considerando un periodo } \tilde{r}_{A}= \\
\text { de } 360 \text { días. }\end{array}$ & $\sqrt{\prod_{a=1}^{A}\left(1+r_{a}\right)}$ & -1 \\
\hline
\end{tabular}

Donde:

$\tilde{r}_{A}$ Rendimiento Anualizado en base al

$r_{a}$ correspondiente a fin de año.

$A$ es el número de años en el periodo

\section{$4 \quad$ Rentabilidad del \\ $5 \quad$ último año máxima \\ 6 Año rentabilidad máxima}

$7 \quad$ Rentabilidad anual mínima

8 Año rentabilidad mínima

$9 \quad$ Desviación media absoluta mensual

10 Desviación estándar mensual

11 Desviación estándar anual

12 Riesgo Anualizado

13 Riesgo Anualizado de Jenssen
Es el rendimiento anual

correspondiente al último año

Es el rendimiento anual máximo en el periodo de

estudio

Es el año que ocurrió la máxima rentabilidad, en el periodo de enero a diciembre de cada año.

Es el rendimiento anual mínimo en el periodo de estudio

Es el año que ocurrió la mínima rentabilidad, en el periodo de enero a diciembre de cada año. Es un indicador estadístico que resume el nivel de variabilidad o dispersión mensual absoluta de los datos respecto al rendimiento promedio. Muestra el nivel de volatilidad mensual en los rendimientos

Muestra en nivel de volatilidad anual en los rendimientos

Es la agregación temporal para determinar la volatilidad anual a partir de la volatilidad mensual. Al riesgo anualizado se incorpora el efecto del valor del dinero en el tiempo $r_{a, \text { Last }}$

$r_{a, \operatorname{Max}}$

$F_{a, \operatorname{Max}}$

$r_{a, \operatorname{Min}}$

$F_{a, \operatorname{Min}}$

$M A D=\frac{\sum_{m=1}^{M}\left|r_{m}-\bar{r}_{M}\right|}{M}$

$\sigma_{M}=\sqrt{\frac{\sum_{m=1}^{M}\left(r_{m}-\bar{r}_{M}\right)^{2}}{M-1}}$

$\sigma_{A}=\sqrt{\frac{\sum_{a=1}^{A}\left(r_{a}-\bar{r}_{A}\right)^{2}}{A-1}}$

$\tilde{\sigma}_{A}=\sigma_{M} \times \sqrt{12}$

$\tilde{\sigma}_{A J}=\sigma_{M} \times \sqrt{12} \times\left(1+\tilde{r}_{A}\right)$ 


\begin{tabular}{|c|c|c|c|}
\hline No. & Indicador & Descripción & Formula \\
\hline 14 & $\begin{array}{l}\text { Coeficiente de } \\
\text { Asimetría }\end{array}$ & $\begin{array}{l}\text { Si el resultado es igual a } 0 \text { la } \\
\text { distribución es simétrica, si es } \\
\text { menor a } 0 \text { la distribución tiene } \\
\text { sesgo negativo, si es mayor a } \\
\text { cero tiene un sesgo positivo. }\end{array}$ & $\begin{array}{l}s=\frac{M}{(M-1)(M-2)} \sum_{m=1}^{M}\left(\frac{r_{m}-\bar{r}_{M}}{\sigma_{M}}\right) \\
\text { Donde: } \\
\text { s es el coeficiente de asimetría }\end{array}$ \\
\hline 15 & $\begin{array}{l}\text { Coeficiente de } \\
\text { Apuntamiento } \\
\text { (Curtosis) }\end{array}$ & $\begin{array}{l}\text { Mide cuán escarpada se } \\
\text { encuentra la distribución, si } \\
\text { es igual a } 3 \text { la distribución es } \\
\text { mesocúrtica, si es mayor a } 3 \\
\text { leptocúrtica, si es menor a } 3 \text { la } \\
\text { distribución es platicúrtica. }\end{array}$ & $\begin{array}{l}c=\left\{\frac{M(M+1)}{(M-1)(M-2)(M-3)} \sum_{m=1}^{M}\left(\frac{m_{m}-\bar{r}_{M}}{\sigma_{M}}\right)^{4}\right\}-\frac{3(M-1)^{2}}{(M-2)(M-3)} \\
\text { Donde: } \\
c \text { es el coeficiente de } \\
\text { apuntamiento }\end{array}$ \\
\hline 16 & Ratio de Sharpe & $\begin{array}{l}\text { Es el gradiente de la Línea } \\
\text { del Mercado de Capitales y } \\
\text { muestra la relación entre el } \\
\text { nivel de recompensa (después } \\
\text { de deducir la tasa libre de } \\
\text { riesgo) respecto al nivel de } \\
\text { riesgo que tiene una inversión. } \\
\text { A mayor gradiente mayor } \\
\text { recompensa por unidad de } \\
\text { rieso. }\end{array}$ & $\begin{array}{l}S R=\frac{\tilde{r}_{A}-r f}{\tilde{\sigma}_{A}} \\
\text { Donde: } \\
S R \text { SR es el Ratio de Sharpe } \\
r f \text { rf es una tasa de interés libre } \\
\text { de riesgo }\end{array}$ \\
\hline 17 & $\begin{array}{l}\text { Máximo descenso } \\
\text { (max drawdown) }\end{array}$ & $\begin{array}{l}\text { Es la máxima pérdida que un } \\
\text { inversionista puede sufrir en } \\
\text { un periodo (comprando en lo } \\
\text { más alto y vendiendo en los } \\
\text { más baio) }\end{array}$ & $D_{\operatorname{Max}}$ \\
\hline 18 & $\begin{array}{l}\text { Mayor descenso } \\
\text { individual (largest }\end{array}$ & $\begin{array}{l}\text { Es la mayor pérdida individual } \\
\text { ininterrumpida en los }\end{array}$ & $D_{\text {lnd }}$ \\
\hline 19 & $\begin{array}{l}\text { individual drawdown) } \\
\text { Descenso promedio } \\
\text { (average drawdown) }\end{array}$ & $\begin{array}{l}\text { rendimientos de la serie. } \\
\text { Es el promedio de los } \\
\text { rendimientos negativos } \\
\text { continuos a lo largo de un } \\
\text { periodo de inversión }\end{array}$ & $\bar{D}=\left|\sum_{j=1}^{j=d} \frac{D_{j}}{d}\right|$ \\
\hline & & & Donde: \\
\hline & & & $\begin{array}{l}D_{j} D_{j} \text { descenso j a lo largo del } \\
\text { periodo }\end{array}$ \\
\hline & & & $\begin{array}{l}d d \text { Número total de descensos en } \\
\text { el periodo }\end{array}$ \\
\hline
\end{tabular}

Fuente: Elaboración propia en base a los indicadores propuestos por Bacon (2013). 


\section{El modelo de mercado y la línea del mercado de títulos}

Un modelo de estas características proporciona una estimación del rendimiento esperado de un título valor o un portafolio de títulos, dados ciertos parámetros. Si el riesgo sistemático es el único parámetro relevante para determinar el retorno, entonces el modelo estimará el rendimiento esperado de cualquier activo en función al nivel de riesgo sistemático.

Conforme establece Jensen (1968), la estimación del desempeño de un portafolio se deriva de una aplicación de los resultados teóricos de los modelos de valoración de activos propuestos en forma independiente por ${ }^{9}$ Sharpe (1964), Litner (1965) y Treynor (1965), también conocido como el modelo

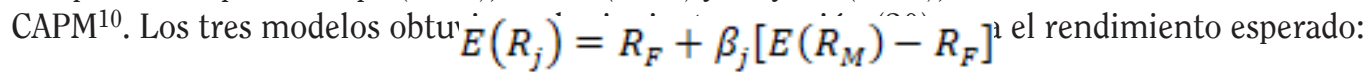

Donde:

$R_{F}$ es la tasa de interés libre de riesgo para un periodo,

$E\left(R_{M}\right)$ es el rendimiento esperado de un periodo para un portafolio de mercar ${ }^{11}$

$B_{j}$ es una medida de riesgo sistemático, que se calcula de la siguiente manera.

$$
\beta_{j}=\frac{\operatorname{cov}\left(R_{j}, R_{M}\right)}{\sigma^{2}\left(R_{M}\right)}
$$

La ecuación (20) señala lo que un título espera ganar dado su nivel de riesgo sistemático $B_{j}$, si el administrador de portafolios o el analista de títulos es capaz de predecir los precios futuros de los títulos valores, entonces será capaz de ganar rendimientos superiores a los implícitos en la ecuación (20) y los riesgos de este portafolio ${ }^{12}$.

Jensen (1968) mostró que el modelo de un periodo puede ser extendido a múltiples periodos, en el cual los inversionistas pueden tener horizontes de inversión heterogéneos donde los títulos son negociados en forma continua en el tiempo, lo que permite generalizar la ecuación (20) en la ecuación (21):

$$
E\left(R_{j t}\right)=R_{F t}+\beta_{j}\left[E\left(R_{M t}\right)-R_{F t}\right]
$$

\footnotetext{
9 Todos estos modelos se basan en los siguientes supuestos: todos los inversionistas son aversos al riesgo y son maximizadores de la utilidad esperada de su riqueza terminal a lo largo de un periodo; todos los inversionistas tienen horizontes de decisión similares y expectativas homogéneas respecto a sus oportunidades de inversión; todos los inversionistas tienen la posibilidad de elegir entre portafolios solamente sobre la base de sus rendimientos esperados y la varianza en sus rendimientos; todos los costos de transacción e impuestos son iguales a cero,; todos los activos son infinitamente divisibles y; los mercados de capitales se encuentran en equilibrio.

10 Por sus siglas en inglés Capital Asset Pricing Model, se trata de un modelo de equilibrio teórico, el cual ha sido contrastado por diversos autores, por ejemplo, varios estudios encontraron que otras variables como el tamaño, el apalancamiento, la relación del valor en libros a valor de mercado también tienen poder de explicación sobre los rendimientos. Sin embargo, una desventaja de estos modelos multifactoriales es que describen en forma ex post los rendimientos y no en forma ex ante como lo hace este modelo. En el presente estudio se eligió esta vía con el propósito de tener una primera aproximación al desempeño de los fondos en Bolivia, que servirá de base para continuar con esta línea de investigación.

11 Este portafolio de mercado consiste en inversiones en cada uno de los activos del mercado en proporción a su fracción sobre el valor del total de activos en el mercado.

12 Como una extensión del modelo para tomar en cuenta, Dumas y Solnik (1995) plantearon el CAPM Internacional (ICAPM), como describen Solnik y McLeavey (2012) en presencia de riesgo de tipo de cambio, se deberán agregar primas de riesgo adicionales en el modelo de valoración de activos, para reflejar la covarianza del activo respecto a los tipos de cambio (las betas de las monedas). Si existen $\mathrm{k}+1 \mathrm{monedas,} \mathrm{existirán} \mathrm{k}$ primas de riesgo adicionales, por tanto, el rendimiento esperado en un activo dependerá de la prima de riesgo de mercado más varias primas de riesgo por cada una de las divisas incluidas en el portafolio, esto se puede apreciar en la ecuación: $E\left(R \_j\right)=R \_F+\beta \_j\left[E\left(R \_M\right)-R \_F\right]+\gamma \_i 1 \square P R D \square \_1+\gamma \_i 2$ $\square$ PRD $\square \_2+\square+\gamma \_$ik $\square$ PRD $\square$ k Donde: $\gamma \_$il a $\gamma \_$ik miden la exposición a las divisas, es decir, miden la sensibilidad de los rendimientos del activo $\mathrm{j}$ expresado en moneda local, frente a las variaciones en el tipo de cambio de las k monedas. $\square$ PRD $\square$ _ 1 a $\square$ PRD $\square$ _k representan la prima de riesgo sobre la divisa k. En el caso de Bolivia en el periodo de 2012 a 2016, de acuerdo a datos oficiales del Banco Central de Bolivia (2017a) el tipo de cambio permaneció constante en 6,86 Bs. por un dólar para la compra; al no existir variación en esta divisa, el coeficiente $\gamma \_$il de esta divisa es igual a cero, por lo que no se incorporan otras variables en (20).
} 
Donde:

t denota un intervalo de tiempo arbitrario con relación a una fecha de inicio y finalización.

Estimando el operador de esperanza matemática en la ecuación (21), mediante la estimación del rendimiento del portafolio de mercado, $y$ dado que la estimación del coeficiente se puede realizar mediante el "Modelo de Mercado"13, Jensen (1968), planteó la siguiente estimación para el rendimiento de un activo $\mathrm{j}^{14}$ :

$$
\tilde{R}_{j t}-R_{F t}=\beta_{j}\left[\tilde{R}_{M t}-R_{F t}\right]+\tilde{e}_{j t}
$$

Donde: $\tilde{e}_{j t}$ es una variable aleatoria, con media cero, homocedástica, con una distribución normal que no tiene una correlación serial.

Como señala Vijay (2013), la implementación más común del modelo de índice de mercado, expuesto en la ecuación (22) es conocido como el Modelo de Mercado (Market Model), en el cual el rendimiento del mercado es el único factor o índice único ${ }^{15}$; en ese sentido, como establece Stapleton y Subrahmanyam ${ }^{16}$ (1983) el modelo se expresa mediante la ecuación ${ }^{17}(23)$ :

$$
\tilde{R}_{j t}=\beta_{0 j}+\beta_{1 j} \tilde{R}_{M t}+\varepsilon_{j t}
$$

Donde:

$\varepsilon_{j t}$ representa un nuevo término de error cuyo valor esperado es igual a cero, con una distribución normal y no tiene una correlación serial.

De acuerdo con Jensen (1968), si el modelo de valoración de activos es válido y si el mercado de capitales se encuentra en equilibrio, la rentabilidad esperada para cualquier activo o portafolio de activos será una función lineal de la cantidad $\beta_{1 j}$, lo que implica que la pendiente de la recta viene dada por la prima de riesgo del mercado $R_{M}-R_{F}$ como se retrata en el gráfico 2, el punto $M$ representa el rendimiento esperado y riesgo del portafolio de mercado, $y$ el punto Rf representa la rentabilidad sobre un activo libre de riesgo. Como menciona Vijay (2013), esta línea se conoce como

13 La medida $\beta$ jde riesgo es aproximadamente igual al coeficiente $\mathrm{b}$ j estimado en el modelo de Jensen.

14 La ecuación (23) puede ser utilizada directamente para realizar una estimación empírica y determinar el riesgo sistemático de cualquier título individual, o también para evaluar el desempeño de aquellos portafolios administrados, como señala Jensen (1968), si el administrador tiene una capacidad superior para realizar pronósticos (quizás porque tiene un conocimiento especial que no está disponible para otros administradores) ,este administrador tenderá a seleccionar de manera sistemática aquellos títulos que le permitan lograr que e j j $>0$ de ahí su portafolio ganará más que la prima de riesgo normal dado su nivel de riesgo. Es posible generar una tolerancia en el modelo, para evaluar la capacidad de pronóstico que tiene un administrador de portafolios, para tal efecto, simplemente sin restringir la estimación de la regresión para que no pase a través del origen. En ese sentido, Jensen planteó la posible existencia de una constante $\alpha$ j no nula en la ecuación, tal y como se muestra en la ecuación: $\mathrm{R}^{\sim} \mathrm{jt}$ - $\mathrm{R} \_\mathrm{Ft}=\alpha \_\mathrm{j}+\beta \_\mathrm{j}$ $\left[\mathrm{R}^{\tilde{L}} \mathrm{Mt}-\mathrm{R} \_\mathrm{Ft}\right]+\tilde{\mathrm{u}} \mathrm{jt}$, Donde: $\alpha \mathrm{t}$ representa la tasa promedio incremental en la rentabilidad del portafolio por unidad de tiempo, $\overrightarrow{\tilde{u}}$ jt $\overrightarrow{\text { representa el }}$ nuevo término de error cuyo valor esperado es igual a cero y no tiene correlación serial.

15 Bajo este modelo, el único factor que explica el comportamiento del rendimiento del activo es el rendimiento del mercado. Como se mencionó previamente, se han desarrollado otros modelos para la valoración de activos; sin embargo, existen varios aspectos que se deben considerar antes de su aplicación, por ejemplo: en los multifactoriales de Fama y French (1993), la formulación es más empírica que teórica, lo que implica una selección rigurosa de las variables, para no incurrir en problemas de multicolinealidad, regresión espuria o endogeneidad; en la teoría de fijación de precios de arbitraje de Ross (1976), la limitación se encuentra en uno de los supuestos, donde señala que los inversores pueden formar carteras bien diversificadas que eliminan el riesgo específico de los activos, este aspecto, no ha sido comprobado en los Fondos de Inversión estudiados; en los modelos con enfoques bayesianos como el propuesto por Black y Litterrman (1991), para su aplicación se requieren valores anteriores (prior values), que para el mercado boliviano no han sido estimados; precisamente los resultados del modelo de un factor podrían utilizarse como prior.

16 En su publicación The Market Model and Capital Asset Pricing Theory: A Note, demostró que el un modelo lineal de Mercado es una condición suficiente para derivar la relación lineal formulada en el CAPM entre $\beta$ y los rendimientos esperados; además, si el rendimiento del mercado tiene una distribución normal la pendiente de la línea del mercado de títulos (Security Market Line) es idéntica a la estimación en media varianza del CAPM, sin embargo, en caso de no cumplirse con esta característica, las diferencias no son mayores. 


\section{GRÁFICO 2 \\ LÍNEA DEL MERCADO DE TÍTULOS}

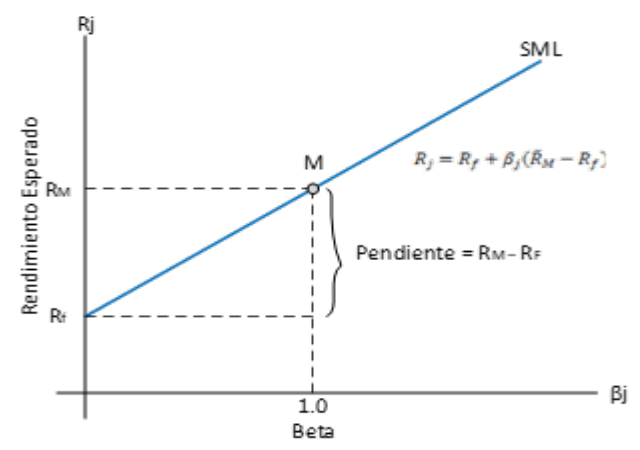

Fuente: Elaboración propia, extraído de Vijay (2013).

\section{RESULTADOS}

La evaluación del desempeño depende en gran medida de las características de los datos utilizados, como menciona Pratt y Grabowski (2010) se deben considerar cuatro factores: la amplitud de tiempo a lo largo del cual los rendimientos históricos son medidos; la periodicidad o frecuencia en la medición de los rendimientos dentro de ese periodo de tiempo; la tasa libre de riesgo por encima de la cual los rendimientos en exceso son calculados y; la selección del índice bursátil para ser utilizado como proxy al mercado.

En el caso de la amplitud de tiempo, siguiendo las directrices del Global Investment Performance Standard (GIPS), se analizó un periodo de 5 años ${ }^{18}$. La periodicidad de los rendimientos puede medirse en forma diaria, semanal, mensual, trimestral o anual. En concordancia con lo descrito por por Pratt y Grabowski (2010) y los trabajos desarrollados por Fama y MacBeth (1973) y Gordon y Roger (1980), se utilizó periodos mensuales.

Respecto a la tasa libre de riesgo, generalmente se utiliza los rendimientos de títulos del tesoro; sin embargo, en el caso de Bolivia no existen publicaciones periódicas sobre la curva de rendimientos; adicionalmente, los datos disponibles presentan variabilidad en el plazo de maduración de estos instrumentos y en su frecuencia de negociación, debido a estas limitaciones, se utilizó como variable proxy de la tasa libre de riesgo R_Ft, la tasa de los depósitos a plazo fijo (DPF) a 30 días ${ }^{19}$, debido a que estas inversiones presentan un nivel de riesgo bajo ${ }^{20}$ y reflejan la dinámica de oferta $y$ demanda que existe en el sistema financiero boliviano ${ }^{21}$.

Del total de 64 fondos, se trabajó con aquellos que contaban con datos sobre sus rendimientos para un periodo de 5 años (desde enero de 2012 hasta diciembre de 2016), haciendo un total de 60 observaciones mensuales. Esto da como resultado una muestra de 36 fondos cuya información se presenta en el siguiente cuadro 5.

\footnotetext{
18 El CFA Institute (2012), en el inciso a, numeral 5.A.1 Presentation and Reporting-Requirement correspondiente al GIPS, señala que como mínimo se deben presentar 5 años de desempeño, sin embargo, la determinación del periodo de estudio también depende de otros elementos relacionados con las condiciones económicas donde se desarrollan este tipo de inversiones. Por ejemplo, un tema relevante a considerar son los ciclos económicos y la liquidez del mercado. En el presente estudio, el periodo de cinco años seleccionado, se caracterizó por la estabilidad macroeconómica del país, reflejada en un crecimiento sostenido del producto interno durante todos los años (con un promedio de 5.29\% anual, medido a través del PIB real), tasas de interés estables y niveles de inflación de un dígito.

19 De acuerdo a datos publicados por el Banco Central de Bolivia (2017b), en el periodo de estudio los DPF en bolivianos tuvieron un rendimiento promedio de $0.27 \%$ anual $y$ de $0.0228 \%$ mensual.

20 Las inversiones en DPF al ser emitidas por instituciones financieras reguladas, se perciben con bajo riesgo crediticio y con alta liquidez.

21 Los DPF se determinan por las entidades de intermediación financiera en función de la liquidez del mercado, estas tasas son al vencimiento.
} 
CUADRO 5

FONDOS DE INVERSIÓN SELECCIONADOS

\begin{tabular}{|c|c|c|c|c|c|}
\hline No. & SAFI & FONDO DE INVERSIÓN & SIGLA & MONEDA & $\begin{array}{l}\text { TIPO DE } \\
\text { FONDO }\end{array}$ \\
\hline 1 & BISA & $\begin{array}{l}\text { A Medida Fondo de Inversión Abierto de Corto } \\
\text { Plazo }\end{array}$ & AME & bolivianos & Abierto \\
\hline 2 & BISA & Ultra Fondo de Inversión Abierto de Mediano Plazo & UFM & bolivianos & Abierto \\
\hline 3 & BNB & Oportuno Fondo de Inversión Corto Plazo & OFI & bolivianos & Abierto \\
\hline 4 & BNB & $\begin{array}{l}\text { En Acción Fondo de Inversión Abierto Mediano } \\
\text { Plazo }\end{array}$ & EAF & bolivianos & Abierto \\
\hline 5 & BNB & Opción Fondo de Inversión Mediano Plazo & $\mathrm{OPU}$ & bolivianos & Abierto \\
\hline 6 & CREDIFONDO & $\begin{array}{l}\text { Credifondo Bolivianos Fono de Inversión Abierto a } \\
\text { Corto Plazo }\end{array}$ & CFB & bolivianos & Abierto \\
\hline 7 & FORTALEZA & $\begin{array}{l}\text { Fortaleza Interés + Fondo de Inversión Abierto } \\
\text { Corto Plazo }\end{array}$ & FOI & bolivianos & Abierto \\
\hline 8 & $\begin{array}{l}\text { MERCANTIL } \\
\text { SC }\end{array}$ & Crecer Bolivianos - Fondo Mutuo Mediano Plazo & $\mathrm{CRB}$ & bolivianos & Abierto \\
\hline 9 & $\begin{array}{l}\text { MERCANTIL } \\
\text { SC }\end{array}$ & Superior Fondo Mutuo Mediano Plazo & SFM & bolivianos & Abierto \\
\hline 10 & $\begin{array}{l}\text { SANTA CRUZ } \\
\text { INVESTMENT }\end{array}$ & $\begin{array}{l}\text { Renta Activa Bolivianos - Fondo de Inversión } \\
\text { Abierto de Corto Plazo }\end{array}$ & $\mathrm{RBF}$ & bolivianos & Abierto \\
\hline 11 & UNIÓN & Fondo de Inversión Dinero Unión - Corto Plazo & DUN & bolivianos & Abierto \\
\hline 12 & UNIÓN & XTRAVALOR Unión FIA Mediano Plazo & XTU & bolivianos & Abierto \\
\hline 13 & BISA & $\begin{array}{l}\text { Capital Fondo de Inversión Abierto de Mediano } \\
\text { Plazo }\end{array}$ & BSK & Dólares & Abierto \\
\hline 14 & BISA & Premier Fondo de Inversión Abierto de Corto Plazo & BSP & Dólares & Abierto \\
\hline 15 & BNB & Efectivo Fondo de Inversión Corto Plazo & EFE & Dólares & Abierto \\
\hline 16 & BNB & Portafolio Fondo de Inversión Mediano Plazo & $\mathrm{PBC}$ & Dólares & Abierto \\
\hline 17 & CREDIFONDO & $\begin{array}{l}\text { Credifondo Renta Fija, Fondo de Inversión Abierto a } \\
\text { Mediano Plazo }\end{array}$ & CFO & Dólares & Abierto \\
\hline 18 & CREDIFONDO & Credifondo Corto Plazo, Fondo de Inversión Abierto & $\mathrm{CCP}$ & Dólares & Abierto \\
\hline 19 & FORTALEZA & $\begin{array}{l}\text { Fortaleza Liquidez Fondo de Inversión Abierto } \\
\text { Corto Plazo }\end{array}$ & FOL & Dólares & Abierto \\
\hline 20 & FORTALEZA & $\begin{array}{l}\text { Fortaleza Porvenir Fondo de Inversión Abierto } \\
\text { Largo Plazo }\end{array}$ & FOP & Dólares & Abierto \\
\hline 21 & FORTALEZA & $\begin{array}{l}\text { Fortaleza Produce Ganancia Fondo de Inversión } \\
\text { Abierto Mediano Plazo }\end{array}$ & PDR & Dólares & Abierto \\
\hline 22 & FORTALEZA & $\begin{array}{l}\text { Fortaleza Renta Mixta Internacional Fondo de } \\
\text { Inversión Abierto Mediano Plazo }\end{array}$ & FRM & Dólares & Abierto \\
\hline 23 & $\begin{array}{l}\text { MERCANTIL } \\
\text { SC }\end{array}$ & Mercantil Fondo Mutuo - Corto Plazo & MFM & Dólares & Abierto \\
\hline 24 & $\begin{array}{l}\text { MERCANTIL } \\
\text { SC }\end{array}$ & $\begin{array}{l}\text { Horizonte Fondo de Inversión Abierto - Mediano } \\
\text { Plazo }\end{array}$ & HOR & Dólares & Abierto \\
\hline 25 & $\begin{array}{l}\text { MERCANTIL } \\
\text { SC }\end{array}$ & Prossimo - Fondo de Inversión Abierto - Corto Plazo & POS & Dólares & Abierto \\
\hline 26 & UNIÓN & Fondo de Inversión Mutuo Unión - Mediano Plazo & UNI & Dólares & Abierto \\
\hline
\end{tabular}




\begin{tabular}{|c|c|c|c|c|c|}
\hline No. & SAFI & FONDO DE INVERSIÓN & SIGLA & MONEDA & $\begin{array}{r}\text { TIPO DE } \\
\text { FONDO }\end{array}$ \\
\hline 27 & FORTALEZA & $\begin{array}{l}\text { Fortaleza Inversión Internacional Fondo de } \\
\text { Inversión Abierto Corto Plazo }\end{array}$ & FII & Dólares & Abierto \\
\hline 28 & $\begin{array}{l}\text { SANTA CRUZ } \\
\text { INVESTMENT }\end{array}$ & $\begin{array}{l}\text { Renta Activa Fondo de Inversión Abierto Corto } \\
\text { Plazo }\end{array}$ & $\mathrm{RAC}$ & Dólares & Abierto \\
\hline 29 & FORTALEZA & Microfinanzas - Fondo de Inversión Cerrado & MIC-A & bolivianos & Cerrado \\
\hline 30 & UNIÓN & PROPYME Unión Fondo de Inversión Cerrado & PUC & bolivianos & Cerrado \\
\hline 31 & FORTALEZA & Impulsor Fondo de Inversión Cerrado & IFI & bolivianos & Cerrado \\
\hline 32 & BISA & $\begin{array}{l}\text { Fondo de Microfinancieras Fondo de Inversión } \\
\text { Cerrado (MICROFIC) }\end{array}$ & MFC & bolivianos & Cerrado \\
\hline 33 & UNIÓN & Proquinua Unión Fondo de Inversión Cerrado & PQU & bolivianos & Cerrado \\
\hline 34 & CAPITAL + & Sembrar Micro Capital Fondo de Inversión Cerrado & $\mathrm{SMC}$ & bolivianos & Cerrado \\
\hline 35 & BISA & Gestión Activa Fondos de Inversión Cerrado & GAC & Dólares & Cerrado \\
\hline 36 & FORTALEZA & $\begin{array}{l}\text { Fondo de Inversión Cerrado Fortaleza Factoring } \\
\text { Internacional }\end{array}$ & FFI & Dólares & Cerrado \\
\hline
\end{tabular}

Fuente: Elaboración propia en base a datos publicados por la ASFI

El análisis se realizó sobre los rendimientos mensuales de cada fondo ${ }^{22}$ expresados en bolivianos. Mediante la aplicación de un test de raíz unitaria de Dickey-Fuller en presencia de outliers aditivos de Vogelsang (1999) se determinó que las series de los fondos GAC, PQU y RAC incluían algunos valores extremos; para dar tratamiento a este fenómeno, mediante el método de Cheung y Lai (1995) se identificaron los periodos correspondientes a estos valores. Posteriormente, siguiendo a Peña, Tiao y Tsay (2001), se realizó una regresión sin constante de estas series respecto a variables dummy con valor de 1 para el periodo correspondiente al valor extremo identificado, cuyos resultados se exponen en Anexo 1. De esta manera, se obtuvieron los residuos que representan a las series consideradas libres de valores extremos.

Nuevamente, se realizaron las pruebas de raíz unitaria a todas las series de tiempo de rendimientos de los fondos seleccionados, cuyos resultados se exponen en el cuadro 6. La primera columna presenta los estadísticos de la prueba Dickey-Fuller aumentado (DFA), la segunda de Phillips Perrón (PP), la tercera muestra el resultado del test DF-GLS y la cuarta columna se encuentra el test KPSS. Como se puede apreciar, con excepción de un caso todas las series resultaron ser estacionarias en niveles.

\footnotetext{
22 Previamente se calculó el valor de cada cuota como el cociente de la cartera neta (expresada en bolivianos) entre el número de cuotas vigentes, como se expuso en el cuadro 4, la tasa de rendimiento diaria de cada fondo se calculó como la tasa de variación del valor cuota del día t-1 al día t. El rendimiento mensual se determinó mediante el producto de los rendimientos obtenidos en los últimos 30 días. Se hace notar que, para el cálculo de estos rendimientos por ganancias de capital, es necesario realizar un ajuste por el pago de dividendos u otros flujos de caja que genera cada instrumento; sin embargo, la información disponible no tiene este ajuste. Esta situación trae como consecuencia, que los rendimientos calculados tengan menor volatilidad, como se puede observar en los resultados que se exponen más adelante.
} 
CUADRO 6

PRUEBAS DE RAÍZ UNITARIA DE LAS SERIES EN NIVELES

\begin{tabular}{|c|c|c|c|c|c|c|c|c|c|}
\hline Variables & DFA & PP & DFGLS & KPSS & Variables & DFA & PP & DFGLS & KPSS \\
\hline OFI & $-2,91$ & $-5,33$ & $-2,59$ & 0,35 & $\mathrm{CFO}$ & $-6,58$ & $-6,97$ & $-1,96$ & 0,68 \\
\hline AME & $-6,56$ & $-6,69$ & $-2,43$ & 0,13 & BSP & $-6,89$ & $-6,97$ & $-6,89$ & 0,11 \\
\hline CRB & $-1,15$ & $-1,44$ & $-0,35$ & 0,77 & $\mathrm{CCP}$ & $-7,12$ & $-7,21$ & $-6,50$ & 0,51 \\
\hline FOI & $-2,75$ & $-5,63$ & $-1,57$ & 0,25 & HOR & $-3,38$ & $-3,23$ & $-3,06$ & 0,10 \\
\hline DUN & $-6,83$ & $-6,72$ & $-6,85$ & 0,07 & POS & $-4,51$ & $-4,60$ & $-2,52$ & 0,09 \\
\hline CFB & $-2,88$ & $-5,59$ & $-2,60$ & 0,58 & FII & $-7,65$ & $-29,36$ & $-6,54$ & 0,15 \\
\hline $\mathrm{RBF}$ & $-6,83$ & $-6,81$ & $-6,64$ & 0,15 & $\mathrm{RAC}$ & $-7,24$ & $-6,08$ & $-1,51$ & 0,13 \\
\hline XTU & $-8,65$ & $-8,77$ & $-1,39$ & 0,08 & FRM & $-6,84$ & $-6,84$ & $-0,33$ & 0,69 \\
\hline EAF & $-11,63$ & $-11,71$ & $-1,01$ & 0,27 & MIC-A & $-5,84$ & $-5,97$ & $-5,73$ & 0,49 \\
\hline $\mathrm{OPU}$ & $-8,16$ & $-8,22$ & $-3,70$ & 0,34 & PUC & $-6,45$ & $-6,45$ & $-1,08$ & 0,27 \\
\hline SFM & $-1,66$ & $-2,42$ & $-1,47$ & 0,65 & IFI & $-8,96$ & $-8,85$ & $-2,21$ & 0,38 \\
\hline UFM & $-6,88$ & $-6,92$ & $-6,73$ & 0,11 & MFC & $-5,77$ & $-5,77$ & $-5,78$ & 0,25 \\
\hline BSK & $-3,11$ & $-6,63$ & $-2,74$ & 0,12 & PQU & $-5,12$ & $-5,05$ & $-3,69$ & 0,57 \\
\hline EFE & $-4,74$ & $-9,32$ & $-0,87$ & 0,19 & SMC & $-7,12$ & $-7,23$ & $-0,50$ & 0,74 \\
\hline FOL & $-10,21$ & $-10,95$ & $-10,30$ & 0,09 & $\mathrm{GAC}$ & $-6,79$ & $-6,79$ & $-6,63$ & 0,18 \\
\hline FOP & $-8,76$ & $-8,97$ & $-8,33$ & 0,10 & FFI & $-6,84$ & $-6,91$ & $-6,89$ & 0,45 \\
\hline MFM & $-4,78$ & $-4,86$ & $-3,22$ & 0,24 & PDR & $-8,04$ & $-8,05$ & $-8,06$ & 0,11 \\
\hline PBC & $-2,66$ & $-9,60$ & $-1,38$ & 0,57 & UNI & $-7,23$ & $-7,23$ & $-5,80$ & 0,25 \\
\hline \multicolumn{5}{|c|}{ Valores críticos } & \multicolumn{5}{|c|}{ Valores críticos } \\
\hline $1 \%$ & $-3,55$ & $-3,55$ & $-2,61$ & 0,74 & $1 \%$ & $-3,55$ & $-3,55$ & $-2,61$ & 0,74 \\
\hline $5 \%$ & $-2,91$ & $-2,91$ & $-1,95$ & 0,46 & $5 \%$ & $-2,91$ & $-2,91$ & $-1,95$ & 0,46 \\
\hline
\end{tabular}

Fuente: Fuente: Elaboración propia.

Para los Fondos RAC, PQU y GAC, se utilizó las series de tiempo libres de valores extremos.

Los gráficos 3, 4 y 5 exhiben las series de tiempo de cada uno de los fondos de inversión. 
GRÁFICO 3

RENDIMIENTO MENSUAL DE LOS FONDOS DE INVERSIÓN ABIERTOS EN BOLIVIANOS
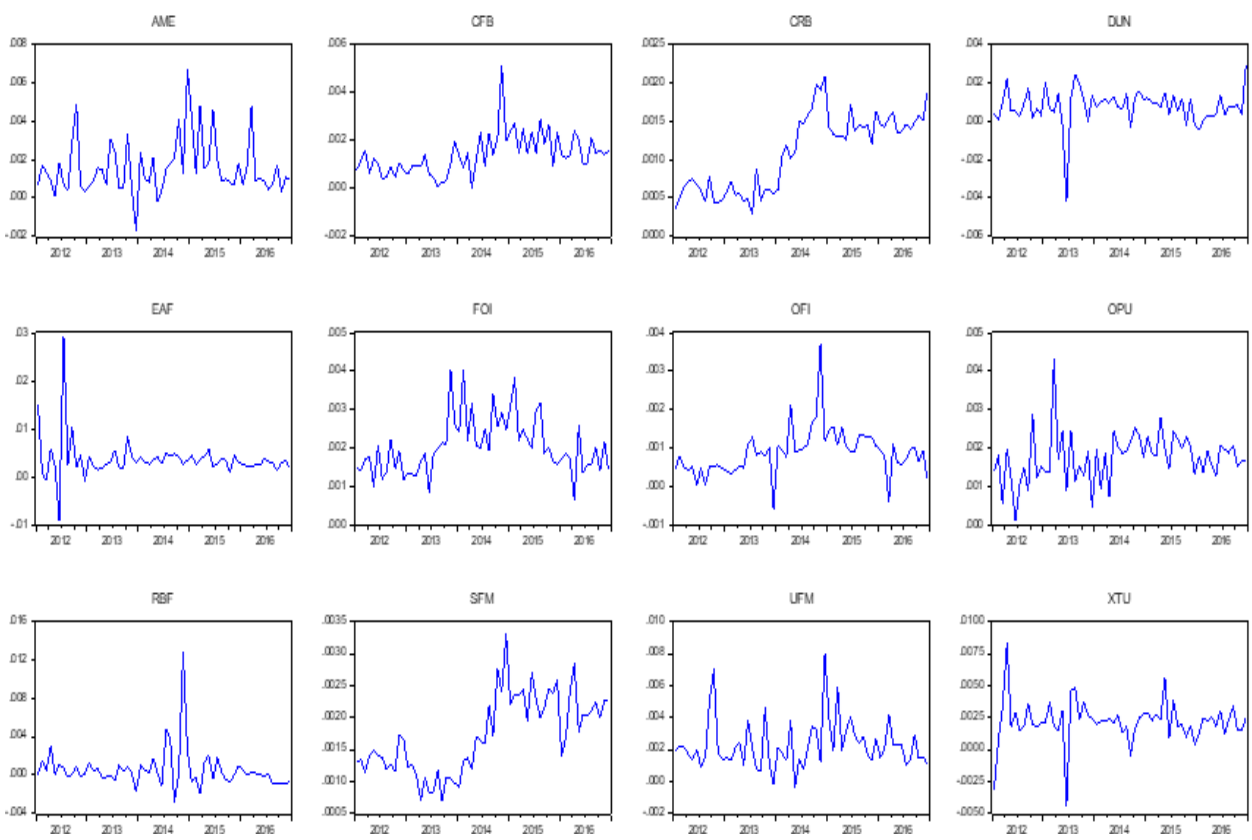

Fuente: Elaboración propia en base a datos obtenidos de la ASFI.

\section{GRÁFICO 4}

RENDIMIENTO MENSUAL DE LOS FONDOS DE INVERSIÓN ABIERTOS EN DÓLARES
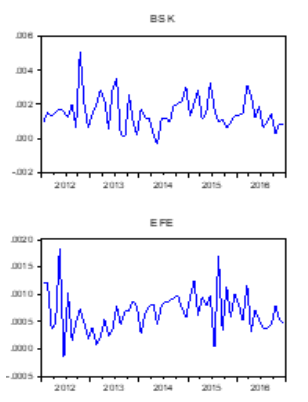

FFu

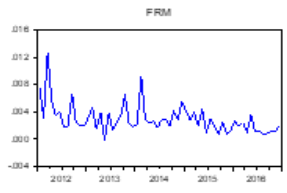

PDF

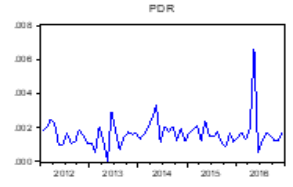

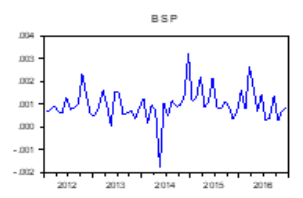

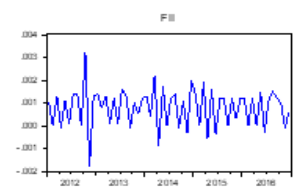

HOR

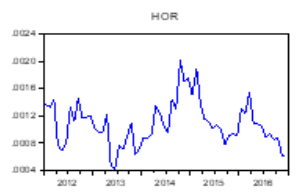

pos

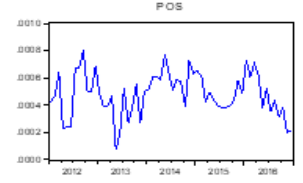

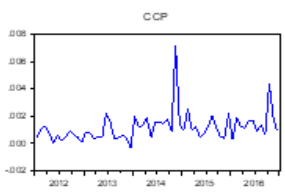

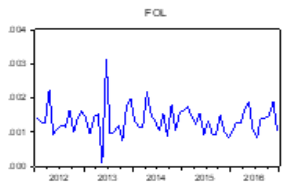

MFM
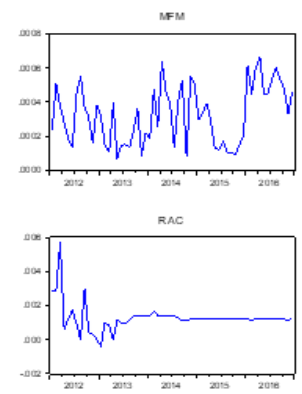
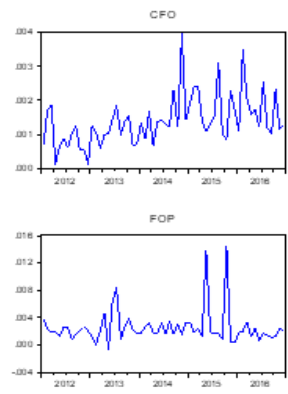

PBC
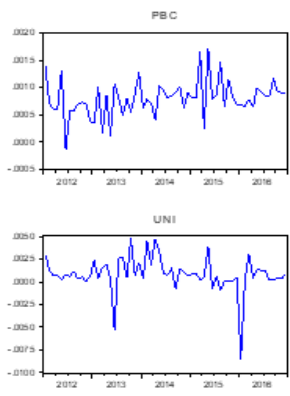

Fuente: Elaboración propia en base a datos obtenidos de la ASFI. 
GRÁFICO 5

RENDIMIENTO MENSUAL DE LOS FONDOS DE INVERSIÓN CERRADOS EN BOLIVIANOS Y EN DÓLARES
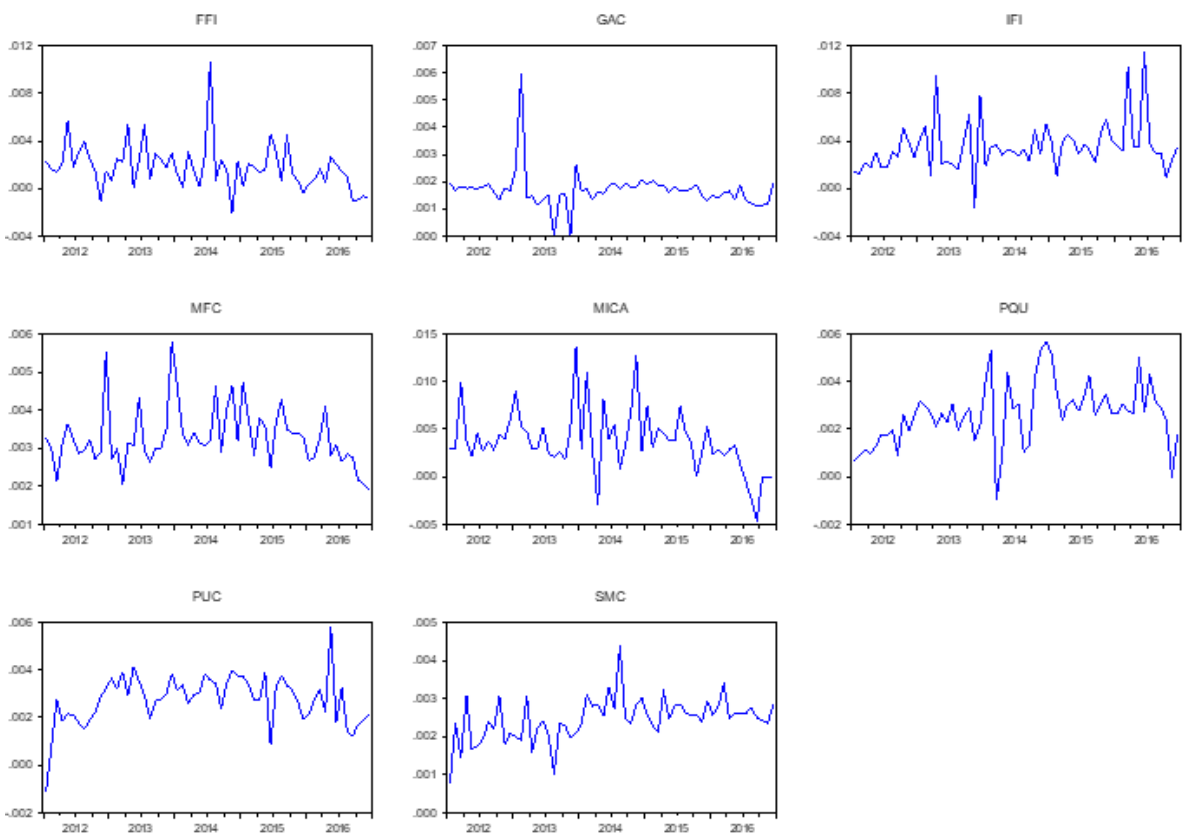

Fuente: Elaboración propia en base a datos obtenidos de la ASFI.

\section{Fondos de Inversión Abiertos en dólares}

En total se analizaron 16 Fondos de Inversión Abiertos (FIA) en dólares, el rendimiento promedio mensual de estos fondos fue de 0,13\%. El fondo FRM alcanzó el máximo rendimiento con 0,34\% seguido del fondo FOP con 0,25\%, ambos de Fortaleza. En cuanto al rendimiento anualizado, el máximo le corresponde al fondo FRM con 3,40\%, mientras el mínimo fue para FII con 0,13\%, los dos de Fortaleza. El promedio de rendimiento anual para todos los fondos fue de 1,44\%.

Los resultados obtenidos por los fondos de inversión, en general, reflejan un bajo nivel de dispersión en la rentabilidad de sus inversiones. El promedio de desviación media absoluta fue de 0,06\%, el Fondo FRM de Fortaleza obtuvo la máxima desviación con 0,20\%. En términos de la desviación estándar de los rendimientos, se realizó una agregación temporal de la desviación estándar mensual que permitió hallar una medida de riesgo anualizada, en este caso, la mayor desviación estándar anual fue para el Fondo FRM de Fortaleza con 1,35\%, mientras que la mínima fue del Fondo POS de Mercantil SC con 0,06\%; se destaca el hecho de que existen 15 Fondos de Inversión que tuvieron una desviación estándar anual menor al 1\%.

Respecto a la distribución de frecuencias de los rendimientos medida, mediante el coeficiente de asimetría y el coeficiente de apuntamiento, el $81 \%$ de los fondos presenta un sesgo positivo y el otro 19\% un sesgo negativo; por otro lado, el 50\% tiene una distribución con alta concentración (distribución leptocúrtica); mientras que el restante 50\% tiene baja concentración (distribución platicurtica). Para contrastar los resultados, se realizó el test de normalidad de Jarque Bera, cuyos resultados señalan que los rendimientos de 6 de los 16 Fondos tienen una distribución normal23 ${ }^{23}$. 
En términos del nivel de recompensa y riesgo medido por el ratio de Sharpe, el promedio fue de 4,27; el resultado máximo fue para el fondo HOR de Mercantil SC con 9,10 y el mínimo fue para FII con -0,50. En cuanto a la caída máxima en los rendimientos del portafolio, el fondo UNI de Unión reportó una caída del $0,88 \%$. Se destaca que 7 fondos no reportaron pérdidas.

Con base en los resultados descritos, se puede concluir que los tres fondos que reportaron mejor desempeño en el periodo de estudio fueron: FOL de Fortaleza, HOR de Mercantil SC y PDR de Fortaleza, mientras que los fondos con los menores resultados fueron FII de Fortaleza, UNI de Unión y MFM de Mercantil SC. Toda esta información se expone en el cuadro 7.

CUADRO 7

INDICADORES DE DESEMPEÑO FONDOS DE INVERSIÓN ABIERTOS EN DÓLARES

\begin{tabular}{|c|c|c|c|c|c|c|c|c|}
\hline $\begin{array}{l}\text { RESUMEN DE } \\
\text { VARIABLES }\end{array}$ & $\begin{array}{l}\text { FORTA } \\
\text { FOL }\end{array}$ & $\begin{array}{l}\text { MSC } \\
\text { HOR }\end{array}$ & $\begin{array}{l}\text { FORTA } \\
\text { PDR }\end{array}$ & $\begin{array}{l}\text { CREDITO } \\
\text { CFO }\end{array}$ & $\begin{array}{c}\text { FORTA } \\
\text { FRM }\end{array}$ & $\begin{array}{l}\text { MSC } \\
\text { MFM }\end{array}$ & $\begin{array}{l}\text { UNION } \\
\text { UNI }\end{array}$ & $\begin{array}{l}\text { FORTA } \\
\text { FII }\end{array}$ \\
\hline $\begin{array}{l}\text { Rendimiento Media } \\
\text { Aritmética Mensual }\end{array}$ & $0,13 \%$ & $0,11 \%$ & $0,17 \%$ & $0,14 \%$ & $0,08 \%$ & $0,05 \%$ & $0,15 \%$ & $0,07 \%$ \\
\hline $\begin{array}{l}\text { Rendimiento Media } \\
\text { Aritmética Anual }\end{array}$ & $1,63 \%$ & $1,31 \%$ & $2,11 \%$ & $1,67 \%$ & $0,97 \%$ & $0,58 \%$ & $1,81 \%$ & $0,80 \%$ \\
\hline $\begin{array}{l}\text { Rendimiento } \\
\text { Anualizado }\end{array}$ & $1,63 \%$ & $1,31 \%$ & $2,11 \%$ & $1,67 \%$ & $0,97 \%$ & $0,58 \%$ & $1,81 \%$ & $0,80 \%$ \\
\hline $\begin{array}{l}\text { Rendimiento anual } \\
\text { último año }\end{array}$ & $150 \%$ & $1,21 \%$ & $2,30 \%$ & $2,00 \%$ & $1,05 \%$ & $0,54 \%$ & $1,63 \%$ & $0,68 \%$ \\
\hline $\begin{array}{l}\text { Rendimiento máximo } \\
\text { anual }\end{array}$ & $1,84 \%$ & $1,54 \%$ & $2,30 \%$ & $2,12 \%$ & $1,16 \%$ & $0,71 \%$ & $2,01 \%$ & $1,01 \%$ \\
\hline Año rendimiento máximo & 2013 & 2014 & 2016 & 2015 & 2015 & 2014 & 2012 & 2015 \\
\hline $\begin{array}{l}\text { Rendimiento mínimo } \\
\text { anual }\end{array}$ & $1,50 \%$ & $0,98 \%$ & $1,91 \%$ & $0,99 \%$ & $0,79 \%$ & $0,45 \%$ & $1,58 \%$ & $0,68 \%$ \\
\hline Año rendimiento mínimo & 2016 & 2013 & 2015 & 2012 & 2012 & 2013 & 2014 & 2013 \\
\hline $\begin{array}{l}\text { Desviación Media } \\
\text { Absoluta mensual }\end{array}$ & $0,03 \%$ & $0,03 \%$ & $0,05 \%$ & $0,05 \%$ & $0,02 \%$ & $0,01 \%$ & $0,07 \%$ & $0,03 \%$ \\
\hline $\begin{array}{l}\text { Desviación Estándar } \\
\text { Muestral mensual }\end{array}$ & $0,04 \%$ & $0,03 \%$ & $0,09 \%$ & $0,07 \%$ & $0,03 \%$ & $0,02 \%$ & $0,10 \%$ & $0,04 \%$ \\
\hline $\begin{array}{l}\text { Desviación Estándar } \\
\text { anual }\end{array}$ & $0,13 \%$ & $0,22 \%$ & $0,19 \%$ & $0,48 \%$ & $0,14 \%$ & $0,10 \%$ & $0,19 \%$ & $0,15 \%$ \\
\hline Riesgo Anualizado & $0,15 \%$ & $0,11 \%$ & $0,30 \%$ & $0,25 \%$ & $0,12 \%$ & $0,06 \%$ & $0,33 \%$ & $0,13 \%$ \\
\hline $\begin{array}{l}\text { Riesgo Anualizado de } \\
\text { Janssen }\end{array}$ & $0,15 \%$ & $0,11 \%$ & $0,30 \%$ & $0,26 \%$ & $0,12 \%$ & $0,06 \%$ & $0,34 \%$ & $0,13 \%$ \\
\hline Sesgo & 0,95 & 0,55 & 3,35 & 1,26 & 0,14 & $-0,24$ & 1,04 & 0,59 \\
\hline Curtosis & 4,12 & 0,47 & 18,72 & 2,36 & 1,28 & $-0,34$ & 2,18 & 1,14 \\
\hline Tasa libre de riesgo & $0,27 \%$ & $0,27 \%$ & $0,27 \%$ & $0,27 \%$ & $0,27 \%$ & $0,27 \%$ & $0,27 \%$ & $0,27 \%$ \\
\hline Ratio de Sharpe & 8,91 & 9,10 & 6,20 & 5,53 & 5,94 & 5,50 & 4,67 & 4,07 \\
\hline Máxima Caída & $0,00 \%$ & $0,00 \%$ & $0,00 \%$ & $0,00 \%$ & $0,01 \%$ & $0,00 \%$ & $0,03 \%$ & $0,02 \%$ \\
\hline $\begin{array}{l}\text { Caída individual más } \\
\text { larga }\end{array}$ & $0,00 \%$ & $0,00 \%$ & $0,00 \%$ & $0,00 \%$ & $0,01 \%$ & $0,00 \%$ & $0,03 \%$ & $0,02 \%$ \\
\hline Caída promedio & $0,00 \%$ & $0,00 \%$ & $0,00 \%$ & $0,00 \%$ & $0,01 \%$ & $0,00 \%$ & $0,03 \%$ & $0,02 \%$ \\
\hline
\end{tabular}


Continuación

\section{CUADRO 7}

INDICADORES DE DESEMPEÑO FONDOS DE INVERSIÓN ABIERTOS EN DÓLARES

\begin{tabular}{|c|c|c|c|c|c|c|c|c|}
\hline $\begin{array}{l}\text { RESUMEN DE } \\
\text { VARIABLES }\end{array}$ & $\begin{array}{l}\text { BISA } \\
\text { BSP }\end{array}$ & $\begin{array}{l}\text { SCINV } \\
\text { RAC }\end{array}$ & $\begin{array}{l}\text { FORTA } \\
\text { FOP }\end{array}$ & $\begin{array}{l}\text { CREDITO } \\
\text { CCP }\end{array}$ & $\begin{array}{l}\text { FORTA } \\
\text { FRM }\end{array}$ & $\begin{array}{l}\text { MSC } \\
\text { MFM }\end{array}$ & $\begin{array}{l}\text { UNION } \\
\text { UNI }\end{array}$ & $\begin{array}{l}\text { FORTA } \\
\text { FII }\end{array}$ \\
\hline $\begin{array}{l}\text { Rendimiento Media } \\
\text { Aritmética Mensual }\end{array}$ & $0,10 \%$ & $0,14 \%$ & $0,25 \%$ & $0,12 \%$ & $0,34 \%$ & $0,03 \%$ & $0,09 \%$ & $0,08 \%$ \\
\hline $\begin{array}{l}\text { Rendimiento Media } \\
\text { Aritmética Anual }\end{array}$ & $1,17 \%$ & $1,51 \%$ & $3,28 \%$ & $1,40 \%$ & $3,40 \%$ & $0,39 \%$ & $0,95 \%$ & $0,13 \%$ \\
\hline Rendimiento Anualizado & $1,17 \%$ & $1,51 \%$ & $3,28 \%$ & $1,40 \%$ & $3,40 \%$ & $0,39 \%$ & $0,95 \%$ & $0,13 \%$ \\
\hline $\begin{array}{l}\text { Rendimiento anual } \\
\text { último año }\end{array}$ & $1,18 \%$ & $1,45 \%$ & $2,19 \%$ & $1,72 \%$ & $2,08 \%$ & $0,62 \%$ & $-0,09 \%$ & $0,06 \%$ \\
\hline $\begin{array}{l}\text { Rendimiento máximo } \\
\text { anual }\end{array}$ & $1,36 \%$ & $1,88 \%$ & $4,49 \%$ & $2,31 \%$ & $4,77 \%$ & $0,62 \%$ & $2,06 \%$ & $0,20 \%$ \\
\hline Año rendimiento máximo & 2015 & 2012 & 2015 & 2014 & 2012 & 2016 & 2014 & 2014 \\
\hline $\begin{array}{l}\text { Rendimiento mínimo } \\
\text { anual }\end{array}$ & $1,05 \%$ & $1,18 \%$ & $2,19 \%$ & $0,69 \%$ & $2,08 \%$ & $0,22 \%$ & $-0,09 \%$ & $0,06 \%$ \\
\hline Año rendimiento mínimo & 2014 & 2013 & 2016 & 2012 & 2016 & 2015 & 2016 & 2016 \\
\hline $\begin{array}{l}\text { Desviación Media } \\
\text { Absoluta mensual }\end{array}$ & $0,05 \%$ & $0,05 \%$ & $0,14 \%$ & $0,07 \%$ & $0,20 \%$ & $0,01 \%$ & $0,11 \%$ & $0,07 \%$ \\
\hline $\begin{array}{l}\text { Desviación Estándar } \\
\text { Muestral mensual }\end{array}$ & $0,07 \%$ & $0,10 \%$ & $0,26 \%$ & $0,11 \%$ & $0,39 \%$ & $0,02 \%$ & $0,19 \%$ & $0,09 \%$ \\
\hline $\begin{array}{l}\text { Desviación Estándar } \\
\text { anual }\end{array}$ & $0,13 \%$ & $0,26 \%$ & $1,02 \%$ & $0,65 \%$ & $1,03 \%$ & $0,16 \%$ & $0,80 \%$ & $0,05 \%$ \\
\hline Riesgo Anualizado & $0,24 \%$ & $0,35 \%$ & $0,89 \%$ & $0,38 \%$ & $1,35 \%$ & $0,06 \%$ & $0,67 \%$ & $0,30 \%$ \\
\hline $\begin{array}{l}\text { Riesgo Anualizado de } \\
\text { Janssen }\end{array}$ & $0,24 \%$ & $0,36 \%$ & $0,92 \%$ & $0,38 \%$ & $1,39 \%$ & $0,06 \%$ & $0,67 \%$ & $0,30 \%$ \\
\hline Sesgo & 0,00 & 3,30 & 3,36 & 3,27 & 4,74 & 0,16 & $-1,97$ & $-0,33$ \\
\hline Curtosis & 4,83 & 13,81 & 13,17 & 15,57 & 28,05 & $-1,20$ & 10,05 & 0,65 \\
\hline Tasa libre de riesgo & $0,27 \%$ & $0,27 \%$ & $0,27 \%$ & $0,27 \%$ & $0,27 \%$ & $0,27 \%$ & $0,27 \%$ & $0,27 \%$ \\
\hline Ratio de Sharpe & 3,76 & 3,50 & 3,38 & 2,98 & 2,32 & 1,92 & 1,02 & $-0,50$ \\
\hline Máxima Caída & $0,17 \%$ & $0,04 \%$ & $0,00 \%$ & $0,03 \%$ & $0,02 \%$ & $0,00 \%$ & $0,88 \%$ & $0,18 \%$ \\
\hline $\begin{array}{l}\text { Caída individual más } \\
\text { larga }\end{array}$ & $0,17 \%$ & $0,04 \%$ & $0,00 \%$ & $0,03 \%$ & $0,02 \%$ & $0,00 \%$ & $0,85 \%$ & $0,18 \%$ \\
\hline Caída promedio & $0,17 \%$ & $0,02 \%$ & $0,00 \%$ & $0,03 \%$ & $0,02 \%$ & $0,00 \%$ & $0,49 \%$ & $0,05 \%$ \\
\hline
\end{tabular}

Fuente: Elaboración propia

\section{Fondos de Inversión Abiertos en bolivianos}

Se analizaron 12 Fondos de Inversión Abiertos (FIA) en bolivianos. El rendimiento promedio mensual de estos fue de 0,17\%, el fondo EAF del BNB alcanzó el máximo rendimiento con 0,36\%, seguido del UFM de BISA con 0,23\%. En cuanto al rendimiento anualizado, el máximo fue alcanzado por EAF de BNB con 4,09\%, el mínimo fue para RBF de Santa Cruz Investment con 0,64\%. El promedio de rendimiento anual para todos los fondos fue de $1,91 \%$. 
El último año el Fondo EAF de BNB obtuvo los mejores resultados con una rentabilidad anual de 3,29\%; en contrapartida, el Fondo RBF de Santa Cruz Investment tuvo la menor rentabilidad anual con $-0,29 \%$.

Los resultados obtenidos por los fondos de inversión en bolivianos reflejan un bajo nivel de dispersión en la rentabilidad de sus inversiones. El promedio de desviación media absoluta fue de 0,08\%, el fondo EAF del BNB obtuvo la máxima desviación con 0,21\%. En términos de la desviación estándar de los rendimientos, se realizó una agregación temporal de la desviación estándar mensual que permitió hallar una medida de riesgo anualizada. En este caso, la mayor desviación estándar anual fue del fondo EAF de BNB con 1,52\%, mientras que la mínima fue del CRB de Mercantil SC con $0,17 \%$. Se destaca el hecho de que existen 11 fondos de inversión que tuvieron una desviación estándar anual menor al $1 \%$.

Respecto a la distribución de frecuencias de los rendimientos cuantificada mediante el coeficiente de asimetría y el coeficiente de apuntamiento, el $83 \%$ de los fondos presenta un sesgo positivo mientras que el $17 \%$ un sesgo negativo. Por otro lado, el $50 \%$ tiene una distribución con alta concentración (distribución leptocúrtica) y el 50\% tiene baja concentración (distribución platicurtica). Para contrastar los resultados, se realizó el test de normalidad de Jarque Bera, cuyos resultados señalan que los rendimientos de 3 de los 12 fondos tienen una distribución normal ${ }^{24}$.

En términos del nivel de recompensa y riesgo medido por el ratio de Sharpe, el promedio fue de 4,63, el resultado máximo fue para el Fondo SFM de Mercantil SC con 8,67 y el mínimo fue para RBF de SANTA CRUZ INVESTMENT con 0,52. En cuanto a la caída máxima en los rendimientos del portafolio, el Fondo DUN de UNIÓN reportó un 0,44\% de caída y el Fondo EAF de BNB tuvo un 0,92\% de caída, 5 fondos no reportaron rendimientos negativos.

Con base en los resultados descritos, se puede concluir que los tres fondos que reportaron mejor desempeño en el periodo de estudio fueron: SFM de Mercantil SC, FOI de Fortaleza y OPU de BNB; mientras que los fondos con los menores resultados fueron RBF de Santa Cruz Investment, DUN de Unión y AME de BISA. Toda esta información se expone en el cuadro 8.

\section{Fondos de Inversión Cerrados en dólares}

Dentro de la muestra estudiada, existen 2 Fondos de Inversión Cerrados (FIC) en dólares, el rendimiento promedio mensual de estos fue de 0,18\%, el Fondo FFI de Fortaleza alcanzó el máximo rendimiento con $0,18 \%$.

En términos de la desviación estándar de los rendimientos, se realizó la agregación temporal de la desviación estándar mensual para hallar una medida de riesgo anualizada. En este caso, la mayor desviación estándar anual fue para el Fondo FFI de Fortaleza con 0,68\%. Para evaluar la distribución de frecuencias de los rendimientos, se aplicó el test Jarque Bera, dando como resultado que ninguna de las series tiene una distribución normal, debido a que ambos fondos presentan sesgo positivo y tienen una distribución con alta concentración (distribución leptocúrtica).

En términos del nivel de recompensa y riesgo medido por el ratio de Sharpe, el promedio fue de 5,62. El resultado máximo fue para el Fondo GAC de BISA con 8,37. En cuanto a la caída máxima en los rendimientos del portafolio, el fondo FFI de FORTALEZA reportó un 0,34\% de caída y GAC de BISA no tuvo pérdidas. Toda esta información se expone en el cuadro 9.

24 Estos Fondos son: SFM, FOI y OPU. 


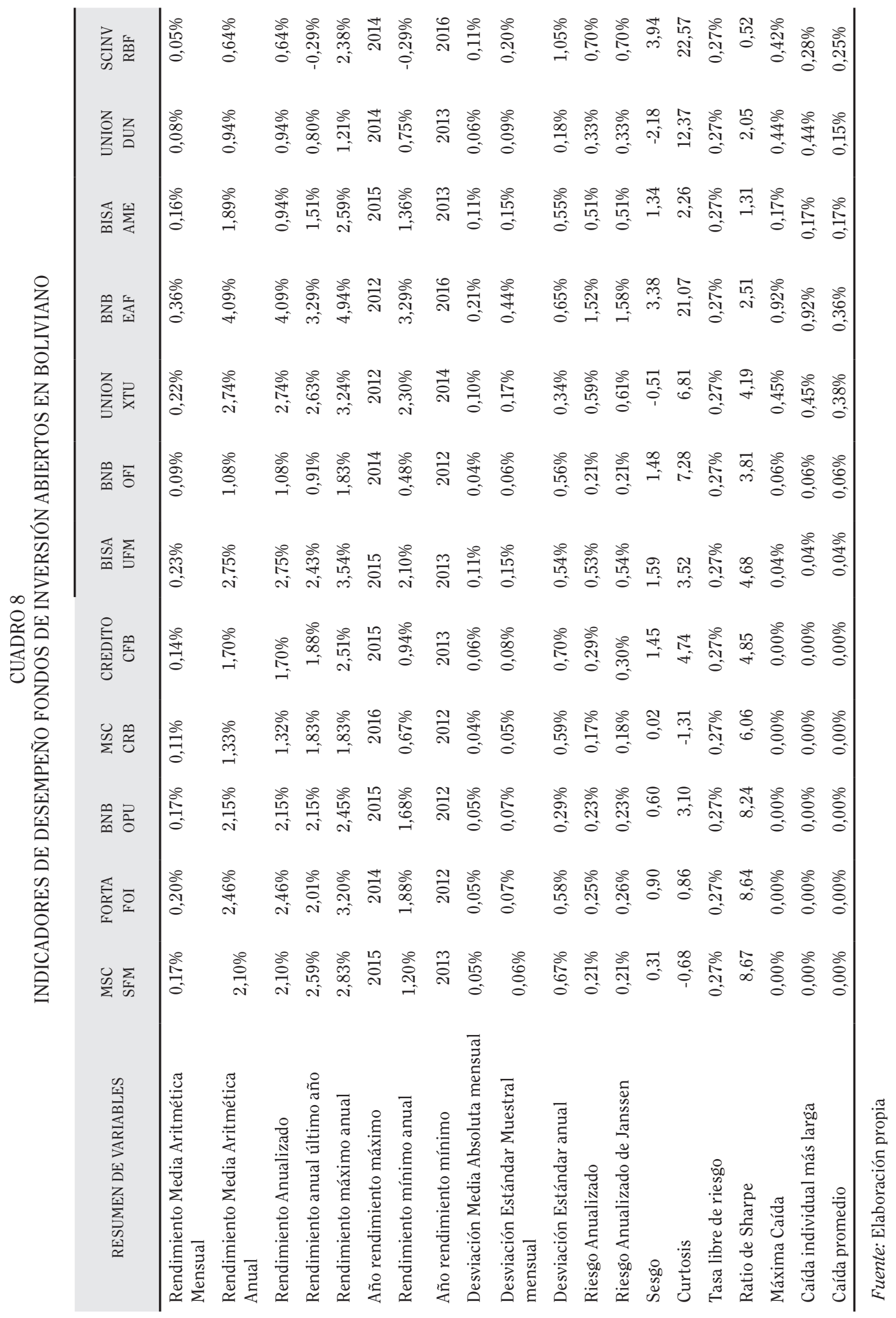


CUADRO 9

INDICADORES DE DESEMPEÑO FONDOS DE INVERSIÓN CERRADOS EN DÓLARES

\begin{tabular}{lrr}
\multicolumn{1}{c}{ RESUMEN DE VARIABLES } & FORTA & \multicolumn{1}{c}{ MSC } \\
& FOL & HOR \\
\hline Rendimiento Media Aritmética Mensual & $0,17 \%$ & $0,18 \%$ \\
Rendimiento Media Aritmética Anual & $2,09 \%$ & $2,23 \%$ \\
Rendimiento Anualizado & $2,09 \%$ & $2,23 \%$ \\
Rendimiento anual último año & $1,74 \%$ & $0,74 \%$ \\
Rendimiento máximo anual & $2,41 \%$ & $3,03 \%$ \\
Año rendimiento máximo & 2013 & 2013 \\
Rendimiento mínimo anual & $1,74 \%$ & $0,74 \%$ \\
Año rendimiento mínimo & 2016 & 2016 \\
Desviación Media Absoluta mensual & $0,03 \%$ & $0,13 \%$ \\
Desviación Estándar Muestral mensual & $0,06 \%$ & $0,20 \%$ \\
Desviación Estándar anual & $0,24 \%$ & $0,88 \%$ \\
Riesgo Anualizado & $0,22 \%$ & $0,68 \%$ \\
Riesgo Anualizado de Janssen & $0,22 \%$ & $0,69 \%$ \\
Sesgo & 5,34 & 1,58 \\
Curtosis & 35,84 & 5,96 \\
Tasa libre de riesgo & $0,27 \%$ & $0,27 \%$ \\
Ratio de Sharpe & $0,00 \%$ & $0,34 \%$ \\
Máxima Caída & $0,00 \%$ & $0,17 \%$ \\
\hline Caída individual más larga & & 2,88 \\
\hline Caída promedio & $0,21 \%$ \\
\hline & & \\
\hline
\end{tabular}

Fuente: Elaboración propia 


\section{Fondos de Inversión Cerrados en bolivianos}

Existen 6 Fondos de Inversión Cerrados (FIC) en bolivianos. El rendimiento promedio mensual de estos fue de 0,31\%. El Fondo MIC-A alcanzó el máximo rendimiento con 0,39\%, seguido del Fondo IFI con $0,35 \%$, ambos de Fortaleza. En cuanto al rendimiento anualizado el máximo fue alcanzado por el fondo MIC-A de Fortaleza con 4,86\%, el mínimo fue para SMC de Capital+ con 2,99\%, el promedio de rendimiento anual para todos los fondos fue de $3,80 \%$.

Los resultados obtenidos por los fondos de inversión también reflejan un bajo nivel de dispersión en la rentabilidad de sus inversiones. El promedio de desviación media absoluta fue de 0,11\%. Los fondos MIC-A e IFI de Fortaleza obtuvieron la máxima desviación con 0,22\% y 0,14\%, respectivamente. En términos de la desviación estándar anual de los rendimientos de los fondos, la mayor fue para fondo MIC-A de Fortaleza con 1,13\%, mientras que la mínima fue SMC de Capital+ con $0,20 \%$. Se destaca el hecho de que existen 5 fondos de inversión que tuvieron una desviación estándar anual menor al 1\%.

Para contrastar los resultados, se realizó el test de normalidad de Jarque Bera, cuyos resultados señalan que los rendimientos de 1 de los 6 fondos (PQU), tienen una distribución normal.

En términos del nivel de recompensa y riesgo medido por el ratio de Sharpe, el promedio fue de 8,60. El resultado máximo fue para los Fondos SMC de Capital+ y MFC de BISA con 13,69 y 13,00 respectivamente. El mínimo fue para MIC-A de Fortaleza con 4,07. En cuanto a la caída máxima en los rendimientos del portafolio, MIC-A de Fortaleza reportó un 0,75\% de caída. Se destaca que 2 fondos no tuvieron caídas en sus rendimientos.

Con base en los resultados descritos, se puede concluir que los tres Fondos que reportaron mejor desempeño en el periodo de estudio fueron: SMC de Capital+, MFC de BISA, y PUC de Unión, mientras que los fondos con los menores resultados fueron MIC-A e IFI de Fortaleza. Toda esta información se expone en el cuadro 10.

CUADRO 10

INDICADORES DE DESEMPEÑO FONDOS DE INVERSIÓN CERRADOS EN BOLIVIANOS

\begin{tabular}{|c|c|c|c|c|c|c|}
\hline RESUMEN DE VARIABLES & $\begin{array}{c}\text { CAPITAL }+ \\
+ \\
\text { SMC }\end{array}$ & $\begin{array}{l}\text { BISA } \\
\text { MFC }\end{array}$ & $\begin{array}{l}\text { UNION } \\
\text { PUC }\end{array}$ & $\begin{array}{l}\text { UNION } \\
\text { PQU }\end{array}$ & $\begin{array}{l}\text { FORTA } \\
\text { IFI }\end{array}$ & $\begin{array}{l}\text { FORTA } \\
\text { MIC-A }\end{array}$ \\
\hline $\begin{array}{l}\text { Rendimiento Media } \\
\text { Aritmética Mensual }\end{array}$ & $0,24 \%$ & $0,33 \%$ & $0,27 \%$ & $0,27 \%$ & $0,35 \%$ & $0,39 \%$ \\
\hline $\begin{array}{l}\text { Rendimiento Media } \\
\text { Aritmética Anual }\end{array}$ & $2,99 \%$ & $3,91 \%$ & $3,38 \%$ & $3,26 \%$ & $4,39 \%$ & $4,87 \%$ \\
\hline Rendimiento Anualizado & $2,99 \%$ & $3,91 \%$ & $3,38 \%$ & $3,25 \%$ & $4,39 \%$ & $4,86 \%$ \\
\hline $\begin{array}{l}\text { Rendimiento anual último } \\
\text { año }\end{array}$ & $3,22 \%$ & $3,19 \%$ & $2,95 \%$ & $3,62 \%$ & $5,32 \%$ & $1,84 \%$ \\
\hline Rendimiento máximo anual & $3,56 \%$ & $4,40 \%$ & $4,04 \%$ & $4,08 \%$ & $5,32 \%$ & $6,09 \%$ \\
\hline Año rendimiento máximo & 2014 & 2014 & 2014 & 2015 & 2016 & 2014 \\
\hline Rendimiento mínimo anual & $2,51 \%$ & $3,19 \%$ & $2,44 \%$ & $1,83 \%$ & $3,58 \%$ & $1,84 \%$ \\
\hline Año rendimiento mínimo & 2012 & 2016 & 2012 & 2012 & 2012 & 2016 \\
\hline $\begin{array}{l}\text { Desviación Media Absoluta } \\
\text { mensual }\end{array}$ & $0,04 \%$ & $0,06 \%$ & $0,08 \%$ & $0,09 \%$ & $0,14 \%$ & $0,22 \%$ \\
\hline
\end{tabular}


Continuación

CUADRO 10

INDICADORES DE DESEMPEÑO FONDOS DE INVERSIÓN CERRADOS EN BOLIVIANOS

\begin{tabular}{|c|c|c|c|c|c|c|}
\hline RESUMEN DE VARIABLES & $\begin{array}{c}\text { CAPITAL }+ \\
+ \\
\text { SMC }\end{array}$ & $\begin{array}{l}\text { BISA } \\
\text { MFC }\end{array}$ & $\begin{array}{l}\text { UNION } \\
\text { PUC }\end{array}$ & $\begin{array}{l}\text { UNION } \\
\text { PQU }\end{array}$ & $\begin{array}{l}\text { FORTA } \\
\text { IFI }\end{array}$ & $\begin{array}{l}\text { FORTA } \\
\text { MIC-A }\end{array}$ \\
\hline $\begin{array}{l}\text { Desviación Estándar } \\
\text { Muestral mensual }\end{array}$ & $0,06 \%$ & $0,08 \%$ & $0,10 \%$ & $0,13 \%$ & $0,22 \%$ & $0,33 \%$ \\
\hline Desviación Estándar anual & $0,46 \%$ & $0,53 \%$ & $0,67 \%$ & $0,88 \%$ & $0,64 \%$ & $1,73 \%$ \\
\hline Riesgo Anualizado & $0,20 \%$ & $0,28 \%$ & $0,36 \%$ & $0,44 \%$ & $0,75 \%$ & $1,13 \%$ \\
\hline $\begin{array}{l}\text { Riesgo Anualizado de } \\
\text { Janssen }\end{array}$ & $0,20 \%$ & $0,29 \%$ & $0,38 \%$ & $0,45 \%$ & $0,78 \%$ & $1,18 \%$ \\
\hline Sesgo & $-0,01$ & 0,85 & $-0,62$ & 0,16 & 1,58 & 0,57 \\
\hline Curtosis & 2,16 & 1,73 & 2,47 & 0,62 & 4,28 & 2,06 \\
\hline Tasa libre de riesgo & $0,27 \%$ & $0,27 \%$ & $0,27 \%$ & $0,27 \%$ & $0,27 \%$ & $0,27 \%$ \\
\hline Ratio de Sharpe & 13,69 & 13,00 & 8,56 & 6,77 & 5,51 & 4,07 \\
\hline Máxima Caída & $0,00 \%$ & $0,00 \%$ & $0,11 \%$ & $0,10 \%$ & $0,16 \%$ & $0,75 \%$ \\
\hline Caída individual más larga & $0,00 \%$ & $0,00 \%$ & $0,11 \%$ & $0,10 \%$ & $0,16 \%$ & $0,46 \%$ \\
\hline Caída promedio & $0,00 \%$ & $0,00 \%$ & $0,11 \%$ & $0,10 \%$ & $0,16 \%$ & $0,52 \%$ \\
\hline
\end{tabular}

Fuente: Elaboración propia

Los resultados generales señalan que los Fondos de Inversión Cerrados obtuvieron mejor desempeño respecto a los Fondos de Inversión Abiertos. En primer lugar, se encuentran los FIC en bolivianos, con un rendimiento promedio de 3,80\% y un ratio de Sharpe de 8,60; en segundo lugar se encuentran los FIC en dólares, con un rendimiento promedio de 2,16\% y un ratio de Sharpe de 5,62; en tercer lugar, los FIA en bolivianos con un rendimiento promedio de 1,91\% con ratio de Sharpe de 4,63; finalmente, los FIA en dólares, con rendimiento promedio de 1,44\% y ratio de Sharpe de 4,27.

\section{Resultados del modelo de mercado}

Para la estimación de los coeficientes del modelo de medición del desempeño expuesto en la ecuación (23), se utilizaron las variables según se explica a continuación:

Como se mencionó previamente, como proxy de la tasa libre de riesgo se utilizó los DPF a 30 días

en bolivianos. Respecto a la rentabilidad del mercado $R_{M t}$, como menciona Vijay (2013) teóricamente el mercado incluye todos los activos riesgosos que incluyen bonos, acciones, bienes raíces, e incluso capital humano; sin embargo, no todos los activos son negociables y no se puede invertir en todos los activos negociables; por tanto, el mercado debería incluir todos los activos posibles. Típicamente un índice de mercado local o regional se utiliza como una proxy para el mercado; sin embargo, una limitación importante para el presente trabajo se debe a que el sistema financiero boliviano y concretamente la Bolsa Boliviana de Valores no cuenta con un índice bursátil de referencia; por tal 
motivo, se optó por realizar una estimación de un portafolio óptimo de mercado en el sentido de Markowitz (1952) ${ }^{25}$, tomando como referencia los 36 fondos de inversión antes mencionados. Este portafolio fue utilizado para representar la rentabilidad del mercado.

El proceso de optimización tuvo como función objetivo la maximización de la relación recompensa - riesgo, tomando como base el trabajo desarrollado por Sharpe (1964), siguiendo el procedimiento descrito por DeFusco, McLeavey, Pinto y Runkle (2015a) y extendiendo la función objetivo utilizada por Vargas (2012). Se determinó como función objetivo el ratio de recompensa respecto a la variabilidad (Reward to Variability Ratio) conocido como ratio de Sharpe ${ }^{26}$, que se expresa mediante la siguiente ecuación:

$$
S=f\left[\left(w_{1} R_{1}+w_{2} R_{2}+\cdots+w_{n} R_{n}\right)\left(w_{1} \sigma_{1}^{2}+w_{2} \sigma_{2}^{2}+\cdots+w_{n} \sigma_{n}^{2}+2 w_{1} w_{2} \sigma_{1,2}+\cdots+2 w_{i} w_{j} \sigma_{i, j}\right)^{-\frac{1}{2}}\right]
$$

Sujeta a las siguientes restricciones:

$$
\sum_{i=1}^{n} w_{i}=1, w_{i} \geq 0
$$

Donde: $w_{i}$ representa el peso o ponderación asignado al fondo i, $R_{i}$ representa la rentabilidad en exceso del fondo i y se calcula como $R_{j t}-R_{F t}, \sigma_{i}^{2}$ es la varianza de los rendimientos de fondo $\mathrm{i}, \sigma_{i, j}$ representa la covarianza entre el fondo i y el fondo $\mathrm{j}$.

Se incorporó una restricción de no negatividad, toda vez que en el mercado boliviano no se pueden realizar operaciones de ventas en $\operatorname{corto}^{27}$, razón por la cual las ponderaciones fueron mayores o iguales a cero, por lo que, al tratarse de una función objetivo no lineal con restricción de no negatividad, como señalan Chiang y Wainwright (2013), fue necesario realizar el proceso de optimización mediante la aplicación de una programación no lineal bajo las condiciones de Kuhn Tucker, para lo cual se planteó la siguiente función Lagrangeana:

$$
Z=f\left(w_{1}, w_{2}, \ldots w_{n}\right)+\lambda\left(1-w_{1}-w_{2}-\cdots w_{n}\right)
$$

Y las condiciones de Kuhn-Tucker aplicadas fueron:

$$
\begin{gathered}
\frac{\partial z}{\partial w_{i}} \leq 0 ; w_{i} \geq 0 \quad y \quad w_{i} \frac{\partial z}{\partial w_{i}}=0 \\
\frac{\partial z}{\lambda} \geq 0 ; \lambda \geq 0 \quad y \quad \lambda \frac{\partial z}{\partial \lambda}=0
\end{gathered}
$$

25 Este enfoque presenta ciertas restricciones: la optimización solo toma en cuenta la media, la varianza y las covarianzas en los datos de entrada; pueden existir fluctuaciones en la frontera eficiente y en la composición del portafolio óptimo, por variaciones en las suposiciones de entrada, que pueden derivar en importantes cambios en los resultados finales; pueden existir soluciones con baja diversificación del portafolio óptimo al estar concentrado en pocos títulos.

26 Considerando que la mayor parte de las series de tiempo no tienen una distribución normal, el presente caso podría extenderse mediante la incorporación en la función objetivo, de los momentos superiores del portafolio como son el co-skewness o co-kurtosis; sin embargo, estos aspectos se encuentran fuera del alcance del presente trabajo.

27 De acuerdo con Investopedia (2017): la venta en corto (del inglés short selling) "es la venta de un valor que no es propiedad del vendedor o que el vendedor ha pedido prestado. La venta en corto está motivada por la creencia de que el precio de un título disminuirá, lo que le permitirá volver a comprarlo a un precio inferior para obtener un beneficio. La venta en corto puede ser provocada por la especulación, o por el deseo de cubrir el riesgo a la baja de una posición larga en la misma garantía o una relacionada". 
Los resultados obtenidos ${ }^{28}$ establecen que el portafolio óptimo de mercado se encuentra compuesto por 18 fondos de inversión, de los cuales 5 son abiertos en bolivianos y 7 abiertos en dólares, 4 cerrados en bolivianos y dos cerrados en dólares. En términos de monedas, aproximadamente el $33 \%$ se encuentra en bolivianos y el $67 \%$ en dólares, como se expone en el gráfico 6 .
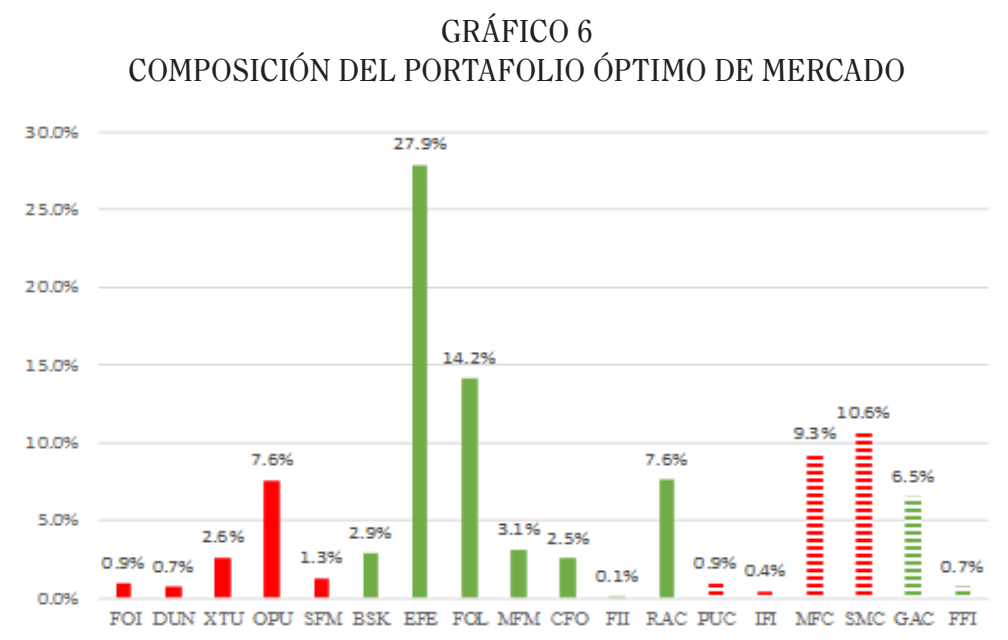

Fuente: Elaboración propia

A partir de la composición del portafolio de mercado, se pudo determinar sus rendimientos históricos en los últimos 5 años, que se presentan en el siguiente gráfico 7.

\section{GRÁFICO 7 \\ RENDIMIENTOS DEL PORTAFOLIO ÓPTIMO DE MERCADO}

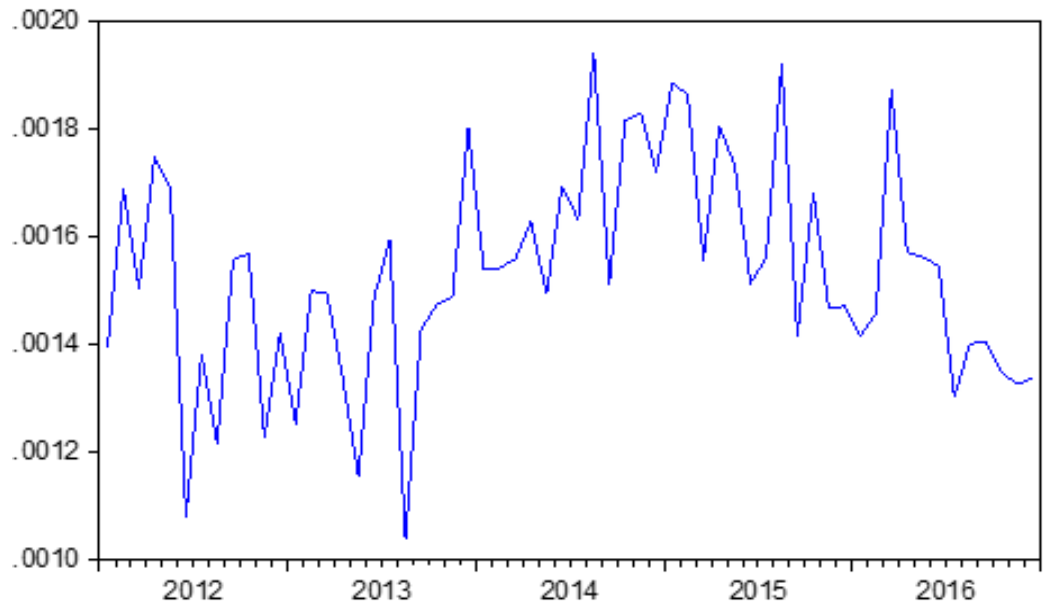

Fuente: Elaboración propia

28 Considerando que en el modelo matemático utilizado no se incluyen otras restricciones, y tomando en cuenta la importancia de la matriz de covarianza (obtenida a partir de los rendimientos históricos) en la solución final, el portafolio óptimo se concentró principalmente en cuatro fondos con aproximadamente el $63 \%$ del total, reduciendo las posibilidades de diversificación. Sin embargo, este portafolio óptimo podrá ser utilizado como punto de referencia, para contrastar resultados con otros métodos de optimización. 
El promedio del rendimiento mensual en bolivianos del portafolio de mercado fue de $0,1530 \%$, con una desviación estándar de 0,0204\% mensual; por otro lado, el test Jarque-Bera señala que los rendimientos tienen una distribución normal y las pruebas de raíz unitaria de Phillips Perrón y KPSS que la serie es estacionaria en niveles ${ }^{29}$.

Con base en los datos obtenidos en la muestra de 36 fondos de inversión, se procedió con la estimación de los coeficientes del modelo expuesto en la ecuación (23). Con el propósito de eliminar los problemas de autocorrelación y heterocedasticidad que impidan realizar inferencias válidas en el modelo, de acuerdo con DeFusco, McLeavey, Pinto y Runkle (2015b), se utilizaron errores estándar robustos mediante la aplicación del estimador de Newey-West. Asimismo, mediante la prueba Ramsey RESET, se comprobó que en 25 de los 36 modelos la forma lineal fue la correcta, se evaluó la especificación del modelo a través del test de endogeneidad de Durbin-Wu-Hausman ${ }^{30}$, comprobando que en 33 de los 36 modelos la variable regresora es exógena, se demostró que el valor esperado de los errores es igual a cero. Finalmente, respecto al supuesto de normalidad en los errores, se identificaron 25 fondos que no cumplen con el mismo, para realizar un contraste más amplio se implementó un procedimiento de bootstraping en los errores. Los resultados permitieron comprobar que con una muestra mayor se cumple el supuesto de normalidad como establece el teorema del límite central y como lo menciona Greene (1999) ${ }^{31}$.

Los resultados muestran que en promedio el coeficiente $R^{2}$ fue de 0,09 , la estimación del coeficiente Beta que mide el riesgo sistemático se encuentra entre 0,030 a 3,017, siendo que, en 25 de los 36 fondos este coeficiente fue estadísticamente significativo.

Para determinar si un fondo se encuentra en equilibrio, se aplicó un test de hipótesis para contraste de medias ${ }^{32}$, a partir de la diferencia entre el rendimiento promedio y el rendimiento requerido calculado a partir de la ecuación $(23)^{33}$. En el Anexo2 se exponen los resultados de la estimación econométrica.

Con base en las estimaciones, en el siguiente gráfico 8 se presenta la dispersión del riesgo de mercado medido a través del coeficiente _ $\mathrm{j}$ en el eje de las abscisas y el rendimiento esperado en bolivianos en eje de las ordenadas. En función de estos datos, se pudo determinar la Línea del Mercado de Títulos (SML) para el mercado boliviano, a partir de la cual se caracterizó a cada uno de los fondos de inversión ${ }^{34}$.

29 El estadístico Jarque Bera tiene un valor de 0,112584 con un p-value de 0,945263, el test estadístico Phillips-Perron tiene un valor de -5,950935 con valor crítico al 0,01 de significación de -3,546099 y el test estadístico de Kwiatkowski-Phillips-Schmidt-Shin tiene un valor de 0,254867 con valor crítico al 0,01 de significación de 0,739 .

30 Se realizó la prueba mediante la formulación de un modelo de mínimos cuadrados en dos etapas y se utilizó como variable instrumental el rendimiento de los DPF a 30 días en bolivianos.

31 El hecho que los residuos no tengan normalidad no tiene un efecto sobre las propiedades de insesgabilidad y eficiencia en los parámetros estimados, el supuesto de normalidad es utilizado para realizar inferencias en los resultados, por esta razón se realizó un bootstraping sobre los errores; sin embargo, esta técnica no permite modificar la forma de la distribución de las variables utilizadas para formular el modelo, las cuales como se mencionó previamente, en varios casos presentan sesgo y leptocurtosis.

32 Se utilizó el siguiente estadístico t de Student: $t_{n-1}=x-\mu / s x / \sqrt{ }$

33 Este cálculo se realizó utilizando el coeficiente $\beta$ de la pendiente, el rendimiento promedio del portafolio de mercado estimado y la proxy de la tasa libre de riesgo.

34 Para tener una mejor representación de la gráfica 9 no se incluyeron los Fondos RBF, FOP y MIC-A. 
GRÁFICO 8

LÍNEA DEL MERCADO DE TÍTULOS PARA LOS FONDOS DE INVERSIÓN EN BOLIVIA

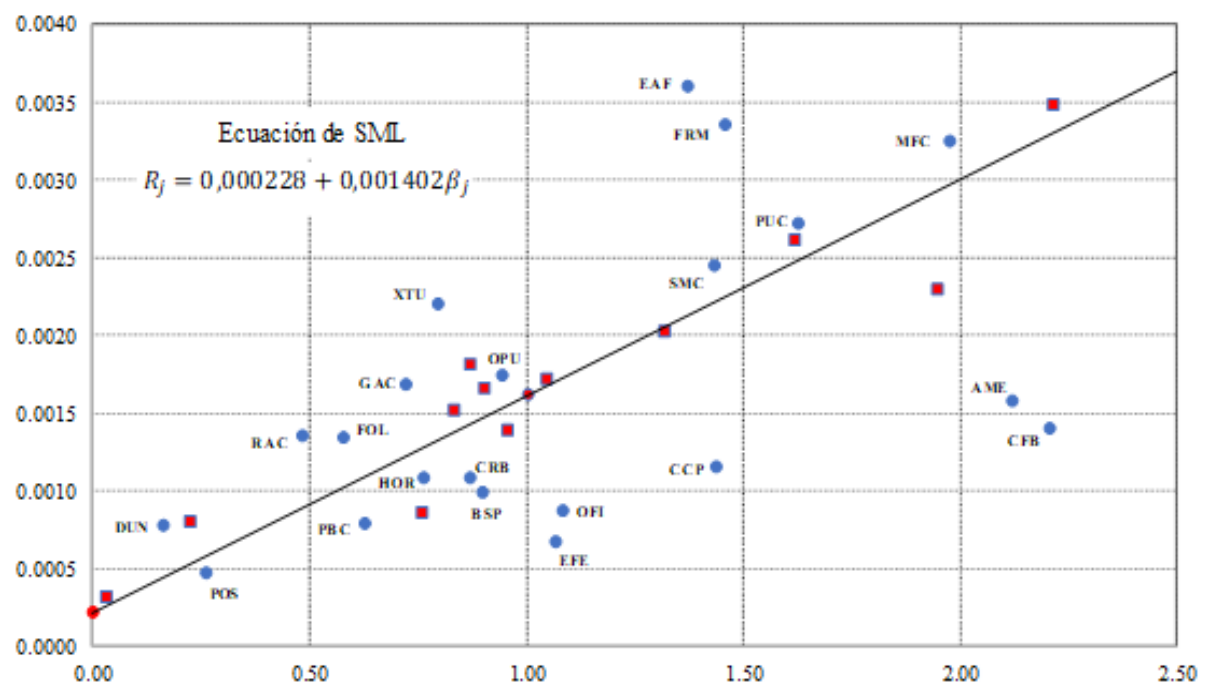

Se agregó la sigla de aquellos Fondos que no se encuentran sobre SML (representados por círculos).

Fuente: Elaboración propia.

Los resultados muestran la existencia de 23 casos en los cuales el nivel de rentabilidad de los fondos de inversión fue diferente a su nivel de equilibrio representado por SML. De este total, 11 obtuvieron rendimientos superiores con una diferencia promedio de $0,06 \%$ mensual equivalente a 0,75\% anual. Por otro lado, 12 obtuvieron un rendimiento menor al requerido con una diferencia promedio de $-0,09 \%$ mensual equivalente a -1,08\% anual. En contrapartida, se encontró que 13 fondos de inversión tienen una relación riesgo-rendimiento que se encuentra sobre SML. Tomando en cuenta estos hallazgos, en Anexo 3 se presenta un resumen de los 10 fondos de inversión que alcanzaron el mejor desempeño en relación al ratio de Sharpe.

\section{CONCLUSIONES}

A lo largo de estos últimos 20 años, desde 1997 hasta 2016, Bolivia experimentó un crecimiento importante en el número de fondos de inversión que participan en su sistema financiero. Con un total de 11 SAFI, se registraron 77 fondos de inversión, de los cuales, a diciembre de 2016 se encontraban vigentes 64 (35 abiertos y 29 cerrados). La etapa de mayor crecimiento se presentó en el periodo desde el 2005 al 2011, cuando se constituyeron un total de 38 nuevos fondos.

La aproximación a la estructura de mercado, mostró que los fondos de inversión expresados en bolivianos se caracterizan por desarrollarse en un mercado con un grado de concentración moderado; sin embargo, los fondos de inversión expresados en dólares se desarrollan en un mercado con un alto grado de concentración, resultados obtenidos mediante la aplicación del Índice Herfindahl-Hirschman.

Se seleccionó aquellos fondos que contaban con datos para un periodo de 5 años desde enero de 2012 hasta diciembre de 2016, obteniendo de esta manera una muestra de 36 fondos. Se realizó la medición del desempeño a partir de información publicada por la ASFI. Los resultados muestran que los Fondos de Inversión Cerrados obtuvieron mejor desempeño respecto a los Fondos de Inversión Abiertos. En primer lugar, se encuentran los FIC en bolivianos, con un rendimiento promedio de 
$3,80 \%$ y un ratio de Sharpe de 8,60; en segundo lugar, los FIC en dólares, con un rendimiento promedio de 2,16\% y un ratio de Sharpe de 5,62; en tercer lugar, los FIA en bolivianos con un rendimiento promedio de 1,91\% con ratio de Sharpe de 4,63; finalmente los FIA en dólares con rendimiento promedio de $1,44 \%$ y ratio de Sharpe de 4,27.

Son 6 las SAFI que se destacan por su participación de mercado y por los resultados alcanzados en sus Fondos de Inversión son: FORTALEZA (FIA en dólares y bolivianos), MERCANTIL SC (FIA en dólares y en bolivianos), BISA (FIC en dólares y bolivianos), BNB (FIA en bolivianos), CAPITAL + (FIC en bolivianos) y UNION (FIC en bolivianos).

Entre las principales limitaciones del trabajo se pueden mencionar: la ausencia de un índice bursátil en el mercado boliviano que pueda ser utilizado como variable proxy del mercado, por lo que se tuvo que construir un portafolio óptimo que está compuesto por 18 fondos; la información disponible para estimar la tasa libre de riesgo, por lo que se utilizó como variable proxy, las inversiones de menor riesgo ofertadas por entidades financieras reguladas.

La estimación del modelo de mercado permitió identificar que 13 fondos mantienen una relación de equilibrio entre la rentabilidad generada y su riesgo sistemático, por lo que se los pudo graficar sobre la Línea del Mercado de Títulos. Sin embargo, existen 23 fondos cuyo rendimiento no es concordante con el nivel de riesgo establecido por el modelo.

Finalmente, a través de la estimación del modelo de mercado, se pudo determinar la rentabilidad esperada para cada uno de los fondos de inversión; sin embargo, el coeficiente de determinación tuvo un valor promedio de 0,09 y en varios casos los parámetros calculados en la regresión no fueron estadísticamente significativos. Esta situación, si bien no resta validez a esta primera contribución para el caso boliviano, plantea la necesidad de proponer nuevas investigaciones orientadas a estudiar las variables proxy que fueron utilizadas $y$ a formular modelos que puedan incluir otras variables que se ajusten a las características particulares de los fondos de inversión en Bolivia.

\section{REFERENCIAS}

Autoridad de Supervisión del Sistema Financiero ASFI. (Octubre de 2017). Evolutivo Fondos de Inversión. Obtenido de https://appweb.asfi.gob.bo/PaginasPublicas2/vistareportevalores/ EvolutivoFondosInversion.aspx

Autoridad de Supervisión del sistema Financiero ASFI. (Abril de 2018). Metodología de valoración. En Recopilación de normas para el mercado de valores (pp. 1-37). Obtenido de http://servdmzw. asfi.gob.bo/CircularValores/Anexos/L08T01/L08T01C01A01.pdf

Bacon, C. (2013). Practical risk-adjusted performance measurement. United Kingdom: Wiley Finance.

Bailey, J., Richards , T., \& Tierney, D. (2016). Reading 31 Evaluating Portfolio Performance. En CFA Institute (Ed.), CFA Level III Volume 6 Trading and Rebalancing, Performance Evaluation, and Global Investment Performance Standards (pp. 120-123). Charlottesville USA: WILEY.

Banco Central de Bolivia BCB. (Octubre de 2017a). Tipo de cambio. Obtenido de https://www.bcb.gob. $\mathrm{bo} / \mathrm{q} \mathrm{q}=$ cotizaciones_tc

Banco Central de Bolivia BCB. (Octubre de 2017b). Tasas de interés. Obtenido de https://www.bcb.gob. bo/?q=tasas_interes

Banco Central de Bolivia BCB. (Octubre de 2017c). Unidad de Fomento a la Vivienda (UFV). Obtenido de https://www.bcb.gob.bo/?q=servicios/ufv/faqs

Black, F., \& Litterman, R. (1991). Global Asset Allocation with Equities, Bonds and Currencies. Fixed Income Research. Goldman Sachs \& Company.

CFA Institute. (2012). Global Investment Performance Standards (GIPS®) Handbook (3 ed.). Charlottesville, USA: CFA Institute. https://doi.org/10.2469/ccb.v2012.n4.full

Cheung, Y.-W., \& Lai, K. S. (1995). Practitioners corner: Lag Order and Critical Values of a Modified Dickey-Fuller Test. Oxford Bulletin of Economics and Statistics, 57(3), 411-419. https://doi. org/10.1111/j.1468-0084.1995.mp57003008.x

Chiang Alpha, C., \& Wainwright, K. (2013). Métodos Fundamentales en Economía Matemática (4º ed.). México: McGrawHill. 
DeFusco, R., McLeavey, D., Pinto, J., \& Runkle, D. (2015a). Reading 53 Portfolio Concepts. En CFA Institute (Ed.), CFA Level II Volume 6 Derivatives and portfolio management (pp. 178-186). Charlottesville, USA: WILEY.

DeFusco, R., McLeavey, D., Pinto, J., \& Runkle, D. (2015b). Reading 10 Multiple Regression and Issues in Regression Analysis. En CFA Institute (Ed.), CFA Level II Volume 1 Ethical and professional standards, quantitative methods, and economics (pp. 188-196). Charlottesville USA: WILEY.

Dumas, B., \& Solnik, B. (1995). The World Price of Foreign Exchange Risk. The Journal of Finance, 50(2), 445-479. https://doi.org/10.2307/2329415

Fama, E., \& French, K. (1993). Common risk factors in the returns on stocks and bonds. Journal of Financial Economics, 33(1), 3-56. https://doi.org/10.1016/0304-405X(93)90023-5

Fama, E. F., \& MacBeth, J. D. (1973). Risk, Return, and Equilibrium: Empirical Tests. Journal of Political Economy, 81(3), 607-636. https://doi.org/10.1086/260061

Gonzales, R. (2011). Riesgo macroeconómico y bolivianización: Un análisis de cointegración con un portafolio dinámico no estacionario de mínima varianza. Revista de Análisis, 15, 9-44.

Gordon J., A., \& Roger D., S. (1980). Consistency of mutual fund performance during varying market conditions. Journal of Economics and Business, 219-226.

Greene, W. (1999). Análisis Econométrico (3 ed.). Madrid: Prentice Hall.

Humerez, J., \& Yañez, E. (2011). Desarrollo del sistema financiero y crecimiento económico, Una aproximación a partir del caso boliviano: 2000-2009. Revista de Análisis, 14, 41-77.

Investopedia. (2017). Short Selling. Obtenido de https://www.investopedia.com/terms/s/shortselling. asp

Jensen, M. (1968). The Performance of Mutual Funds in the Period 1945-1964. The Journal of Finance, 23(2), 389-416. https://doi.org/10.1111/j.1540-6261.1968.tb00815.x

Lintner, J. (1965). Security prices, risk, and maximal gains from diversification. The Journal of Finance, 20(4), 587-615. https://doi.org/10.1111/j.1540-6261.1965.tb02930.x

Markowitz, H. (1952). Portfolio selection. The Journal of Finance, 7(1), 77-91. https://doi. org/10.1111/j.1540-6261.1952.tb01525.x

Peña, D., Tiao, G., \& Tsay, R. (2001). A Course in Time Series Analysis. Nueva York: John Wiley \& Sons Inc.

Pratt, S., \& Grabowski, R. (2010). Cost of capital. Cost of Capital: Workbook and Technical Supplement ( $4^{\circ}$ ed.). California: Wiley.

Ross, S. (1976). The arbitrage theory of capital asset pricing. Journal of Economic Theory, 13(3), 341360. https://doi.org/10.1016/0022-0531(76)90046-6

SAFI BISA. (Abril de 2018). ¿Qué es un fondo de inversión?. Obtenido de http://www.bisasafi.com/ que-es-un-fondo-de-inversion

Sharpe, W. F. (1964). Capital Asset Prices: A Theory of Market Equilibrium under Conditions of Risk. The Journal of Finance, 19(3), 425-442. https://doi.org/10.2307/2977928

Solnik, B., \& Mcleavey, D. (2012). International Asset Princing. En CFA Institute (Ed.), CFA Level II Volume 6, Derivatives and Portfolio Management (pp. 489-497). Pearson.

Stapleton, R. C., \& Subrahmanyam, M. G. (1983). The Market Model and Capital Asset Pricing Theory: A Note. The Journal of Finance, 38(5), 1637-1642. https://doi.org/10.1111/j.1540-6261.1983. tb03846.x

Treynor, J. (1965). How to Rate Management of Investment Funds. Harvard Business Review, XLIII, 63-75.

Vargas, A. (2012). Gestión Activa de Portafolios mediante la aplicación del modelo de Treynor-Black. Revista Investigación \& Desarrollo, 12(1), 17-32. https://doi.org/10.23881/idupbo.012.1-2e

Vijay, S. (2013). Portfolio Risk and Return: Part II. En CFA Institute (Ed.), CFA Level I Volume 4, Corporate Finance and Portfolio Management (pp. 326-365). Charlottesville USA: WILEY.

Vogelsang, T. J. (1999). Two Simple Procedures for Testing for a Unit Root When There are Additive Outliers. Journal of Time Series Analysis, 20(2), 237-252. https://doi.org/10.1111/14679892.00135 
ANEXO 1

RESULTADOS DE LA REGRESIÓN PARA OBTENER SERIES LIBRES DE VALORES EXTREMOS

\begin{tabular}{|c|c|c|c|c|c|c|c|c|}
\hline $\begin{array}{c}\text { Variable } \\
\text { Dependiente }\end{array}$ & $\begin{array}{c}\text { Variables } \\
\text { Independientes }\end{array}$ & Coeficiente & $\begin{array}{l}\text { Error } \\
\text { Estándar }\end{array}$ & Estadístico t & Probabilidad & $\begin{array}{l}\text { S.E. de la } \\
\text { regresión }\end{array}$ & $\begin{array}{c}\text { Suma } \\
\text { de los } \\
\text { residuos al } \\
\text { cuadrado }\end{array}$ & $\begin{array}{c}\text { Log } \\
\text { likelihood }\end{array}$ \\
\hline \multirow[t]{3}{*}{ Fondo GAC } & $\begin{array}{r}\text { Dummy } 1 \text { (valor } 1 \text { en } \\
\text { agosto 2013) }\end{array}$ & $-0,0041$ & 0,0017 & $-2,3909$ & 0,0201 & 0,0017 & 0,0002 & 304,1659 \\
\hline & $\begin{array}{r}\text { Dummy } 2 \text { (valor } 1 \text { en } \\
\text { noviembre } 2013 \text { ) }\end{array}$ & $-0,0096$ & 0,0017 & $-5,6437$ & 0,0000 & & & \\
\hline & $\begin{array}{r}\text { Dummy } 3 \text { (valor } 1 \text { en } \\
\text { febrero } 2013 \text { ) }\end{array}$ & 0,0060 & 0,0017 & 3,5264 & 0,0008 & & & \\
\hline Fondo PQU & $\begin{array}{r}\text { Dummy } 1 \text { (valor } 1 \text { en } \\
\text { noviembre } 2016 \text { ) }\end{array}$ & $-0,0463$ & 0,0029 & $-15,7197$ & 0,0000 & 0,0029 & 0,0005 & 269,3681 \\
\hline \multirow[t]{2}{*}{ Fondo RAC } & $\begin{array}{r}\text { Dummy } 1 \text { (valor } 1 \text { en } \\
\text { agosto 2012) }\end{array}$ & $-0,0165$ & 0,0017 & $-9,5834$ & 0,0000 & 0,0017 & 0,0002 & 302,5666 \\
\hline & $\begin{array}{r}\text { Dummy } 2 \text { (valor } 1 \text { en } \\
\text { diciembre } 2012 \text { ) }\end{array}$ & $-0,1314$ & 0,0017 & $-76,1714$ & 0,0000 & & & \\
\hline
\end{tabular}

Fuente: Elaboración propia 


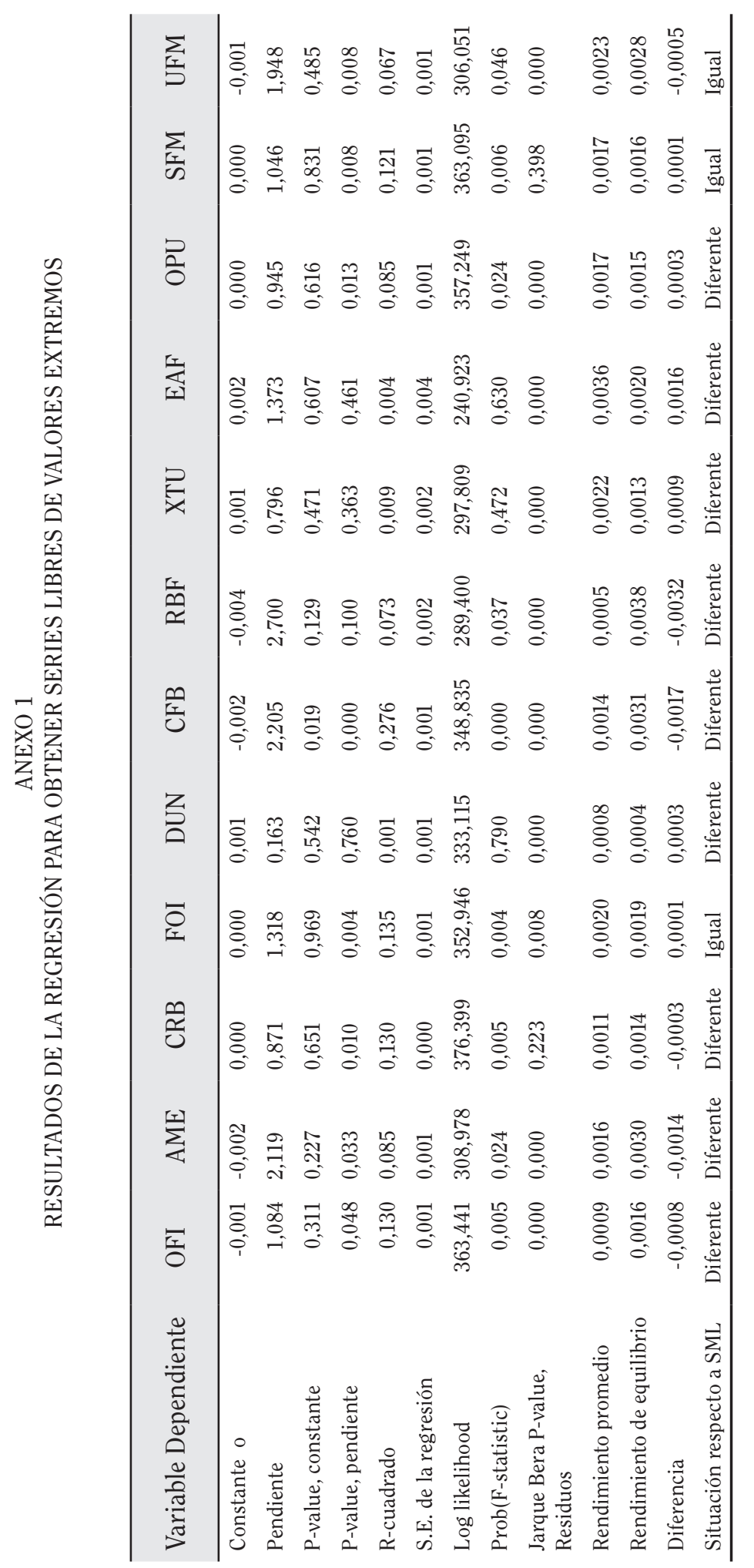

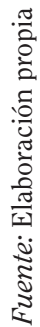




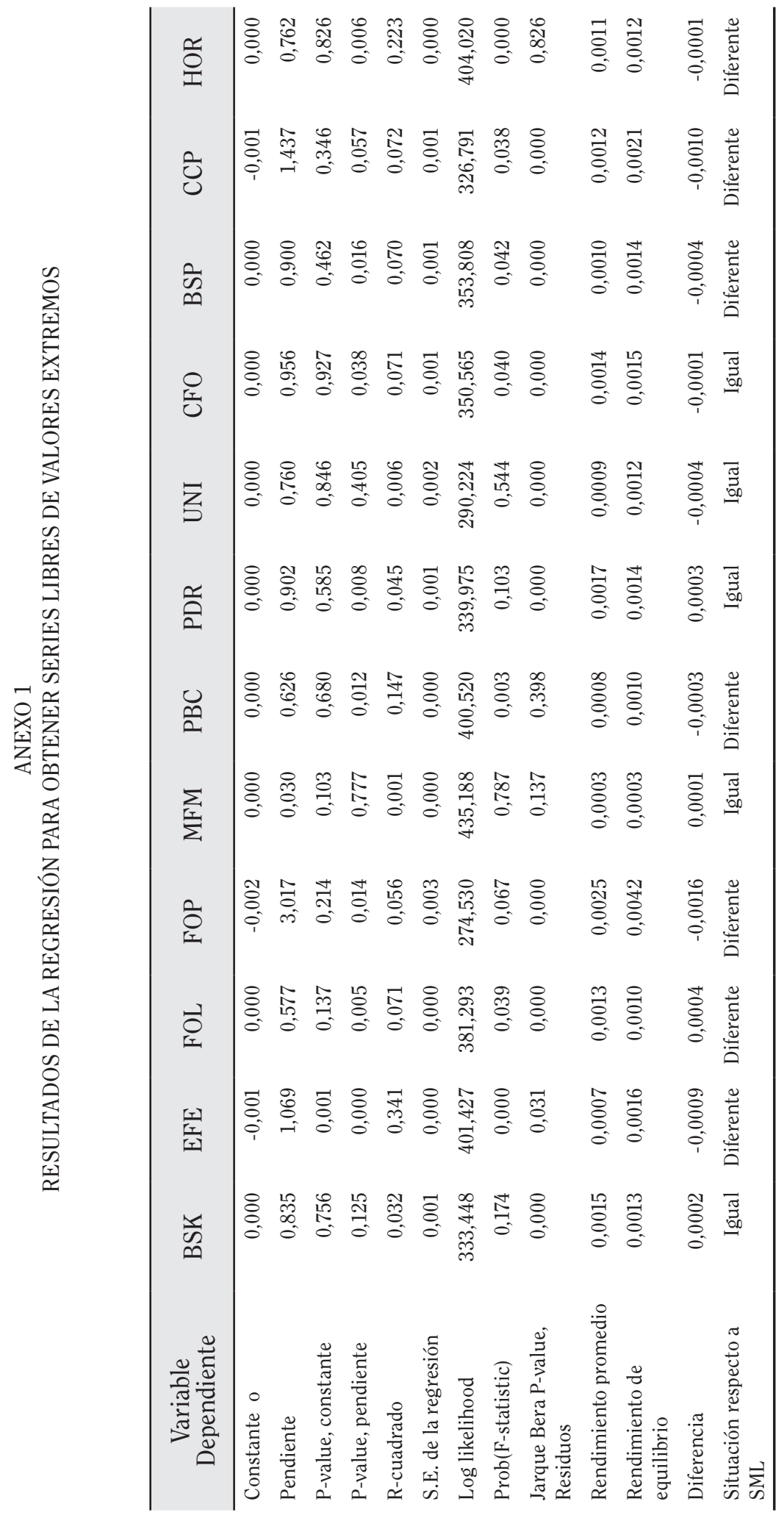




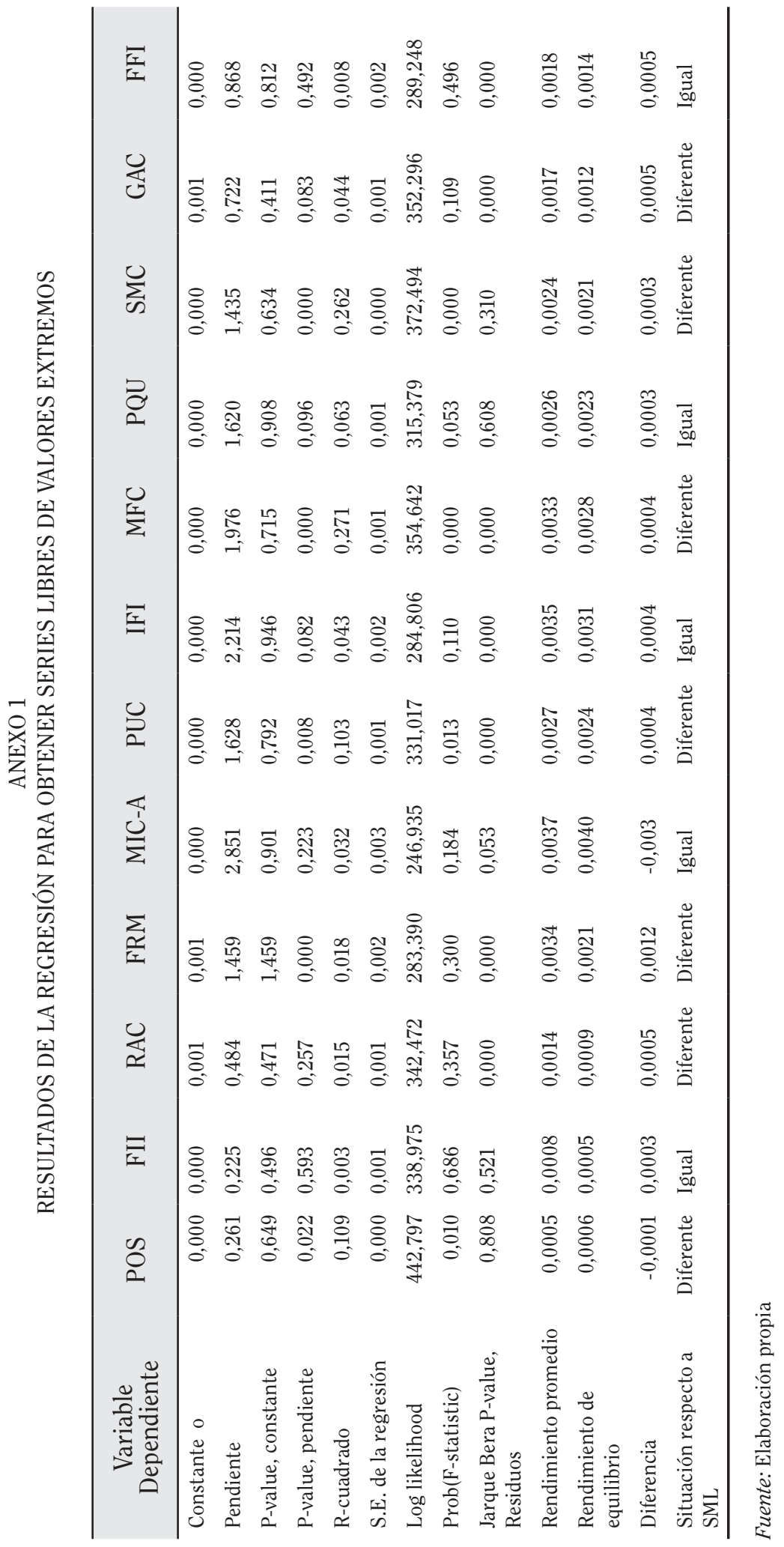




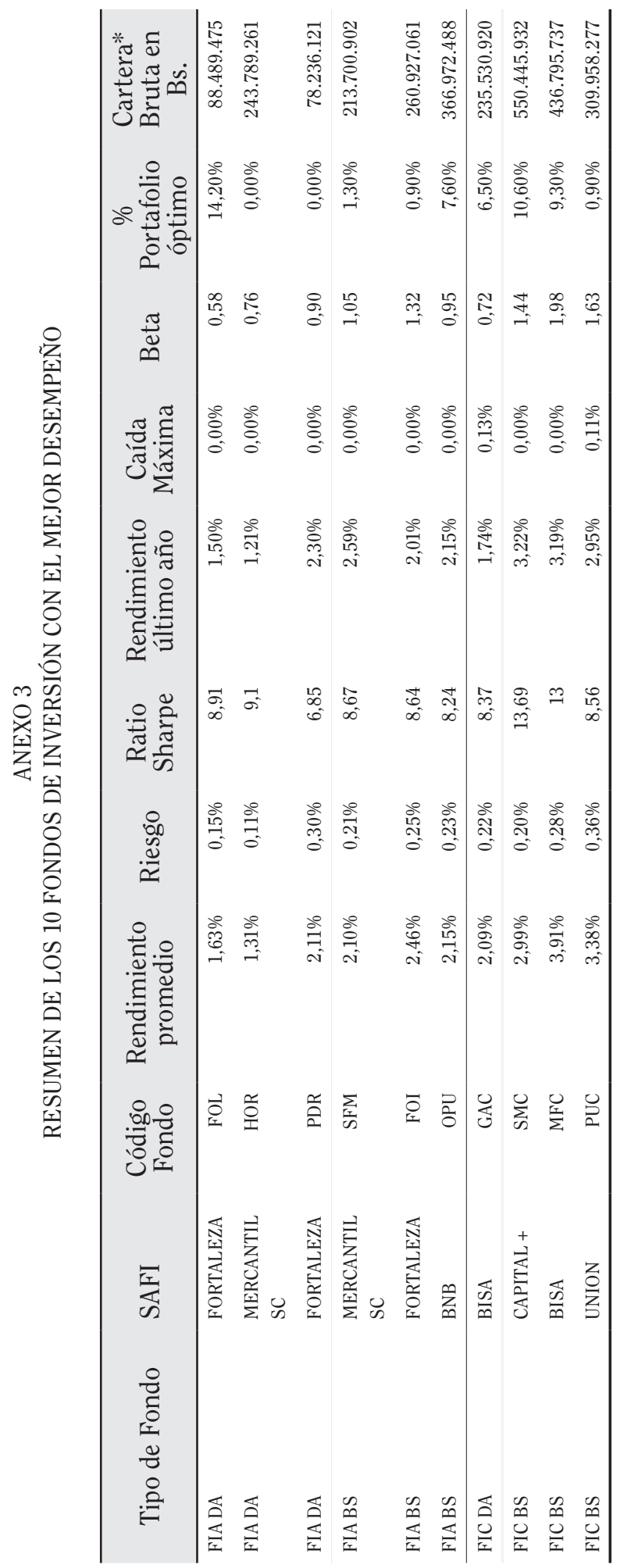

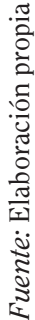

